\title{
Efficient multi-scale imaging of subsurface resistivity with uncertainty quantification using Ensemble Kalman Inversion
}

Chak-Hau Michael Tso ${ }^{1,4}$, Marco Iglesias ${ }^{2}$, Paul Wilkinson ${ }^{3}$, Jonathan Chambers ${ }^{3}$ and Andrew Binley ${ }^{4}$

1 UK Centre for Ecology and Hydrology, Lancaster, UK

${ }^{2}$ Department of Mathematical Sciences, University of Nottingham, UK

3 British Geological Survey, Keyworth, UK

${ }^{4}$ Lancaster Environment Centre, Lancaster University, UK

corresponding authors: mtso@ceh.ac.uk

SUMMARY

Electrical resistivity tomography (ERT) is widely used to image the Earth's subsurface and has proven to be an extremely useful tool in application to hydrological problems. Conventional smoothness-constrained inversion of ERT data is efficient and robust, and consequently very popular. However, it does not resolve well sharp interfaces of a resistivity field and tends to reduce and smooth resistivity variations. These issues can be problematic in a range of hydrological or near-surface studies, e.g. mapping regolith-bedrock interfaces. While fully Bayesian approaches, such as those employing Markov chain Monte Carlo sampling, can address the above issues, their very high computation cost make them impractical for many applications. Ensemble Kalman Inversion (EKI) offers a computationally efficient alternative by 
approximating the Bayesian posterior distribution in a derivative-free manner. We propose a new EKI-based framework for ERT which estimates a resistivity model and its uncertainty at a modest computational cost. Our EKI framework uses a level set parameterization of the unknown resistivity to allow efficient estimation of discontinuous resistivity fields. Instead of estimating level set parameters directly, we introduce a second step to characterize the spatial variability of the resistivity field and infer length scale hyper-parameters directly. We demonstrate these features by applying the method to a series of synthetic and field examples. We also benchmark our results by comparing them to those obtained from standard smoothnessconstrained inversion. Resultant resistivity images from EKI successfully capture arbitrarily shaped interfaces between resistivity zones and the inverted resistivities are close to the true values in synthetic cases.

Key words: Ensemble Kalman methods - ERT - inversion - data assimilation uncertainty quantification - level sets.

\section{INTRODUCTION}

Electrical resistivity imaging (ERI), or electrical resistivity tomography (ERT), is an effective method to reveal the subsurface structure of the Earth's near-surface. It can provide a proxy of properties of interest at spatial coverage and resolution that are not attainable by pointbased sampling methods. When used in a time-lapse manner, ERT surveys can be repeated frequently to capture changes in the resistivity distribution, offering insight into subsurface processes. Recent advances in instrumentation allows the deployment of ERT surveys with a large number of electrodes and the collection of time-lapse data using autonomous systems. ERT is widely used in a large number of environmental and engineering applications, such as hydrological characterization, landslide monitoring, studying root water uptake, and archaeological exploration. Many applications require the identification of boundaries between two or more subsurface zones and reliable estimates of resistivity within each zone (e.g. soil horizons, geological facies, engineered structures, or wetting fronts). Moreover, some measures of uncertainty to these estimates are often desirable to facilitate interpretation and decision making.

Smoothness constrained inversion (e.g. Binley 2015) is the most commonly used method 
for ERT inversion. Although it is an efficient and robust method, it explicitly favors the smoothest resistivity field (by $L^{2}$ measure)that honors the observed data, subject to prescribed constraints. In the presence of sharp changes of resistivity in the subsurface, the interfaces between regions are often not clearly resolved. Moreover, the inverted resistivity values tend to be in the region of the mean (linear or log) resistivity of the entire domain, rather than in the range of the actual resistivities in each zone. This then limits our ability to use the inverted resistivities to map directly to hydrological models via petrophysical transforms. While these issues can be alleviated partly using minimum support functionals (e.g. Nguyen et al. 2016) or parameter disconnect if the boundaries are known (e.g. Slater \& Binley 2003; Johnson et al. 2012), a general approach is lacking to image the subsurface with arbitrarily shaped zones. There exist approaches to derive zonal or facies membership from smooth inverted images, mainly post-possessing the images via clustering or edge detectors (Chambers et al. 2014; Ward et al. 2014), or deriving conditional probability maps from co-located measurements from direct sampling (Hermans \& Irving 2017). However, their performance depends on the results of the smoothness-constrained inversion results. Smoothness constrained inversion is also not particularly well-suited for uncertainty quantification, especially when there are sharp boundaries. Conducting uncertainty analysis on the smoothly varying fields allows one to understand their variability, but provides little information on the location of potential discontinuities in the actual resistivity field.

Incorporation of geostatistical information can greatly improve inversions. Early attempts include the sequential successive linear estimator (SSLE, Yeh et al. 2002) (see also a comparison between smoothness-constrained and geostatistical inversion by Englert et al. (2016)). More recent attempts include applying the principal component geostatistical inversion on ERT data (Kitanidis 2015). Geostatistical approaches have largely been based on variograms and they assume a stationary random field. This assumption is violated in systems where there are features of strikingly different orientation and abrupt changes or discontinuities such as those arising from stratified geologic features. Recent methods have taken advantage of the nonstationary Matérn family of covariance functions, where variogram and spatial scales are estimated at each location in the model domain as a stochastic process. Such method has been used increasingly for spatial modeling in geophysics and precipitation modelling and was first used in 2D Bayesian ERT inversion by Bouchedda et al. (2017).

With increasing interest in quantifying the uncertainty in ERT estimates and improving the identification of features (Linde et al. 2017; Andersen et al. 2003; Ramirez et al. 2005; Irving \& Singha 2010), there has been significant interest in using fully Bayesian approaches 
based on Markov Chain Monte Carlo (MCMC) to approximate the posterior probability density function (pdf) of the unknown resistivity field (i.e. posterior of resistivity at each pixel). Accurate approximations of the full posterior pdf are useful for quantifying the uncertainty of all possible modelling outcomes emerging from ERT. Recent work includes the trans-dimensional inversion concept where the number and density of parameters are estimated alongside the parameter values (Galetti \& Curtis 2018), which adaptively avoids overor under- parametrizing the given inverse problem. A related approach uses a fixed parameter mesh and in each MCMC iteration, the estimates of the interface between the two zones (i.e. a polyline) are first updated, followed by estimates of the parameter fields within each sub-domain (de Pasquale et al. 2019). Similarly, to speed up the generation of MCMC proposals and hence convergence, an area-to-point kriging approach has been proposed recently to generate fine-scale multi-Gaussian realizations from smooth tomographic images obtained from smoothness-constrain inversions (Nussbaumer et al. 2019).

The theory of fully Bayesian methods such as MCMC ensures that sampling approximations converge to the target (posterior) distribution. In practice, however, it is widely known that these methods require hundreds of thousands or even millions of model runs to produce accurate approximations. A few thousands of MCMC samples can, indeed, be used to approximate regions of high-probability. However, regions of low probability under the posterior pdf curve can be under-sampled since most MCMC proposals are based on local-moves which tend to reject samples that belong in those low probability regions. It comes as no surprise that most fully Bayesian methods in the context of ERT have been only applied to 2D settings and/or focus on approximating only the high probability areas which could, perhaps, be approximated more cost-effectively with methods based on Gaussian approximations such as the one that we introduce in this work.

Data assimilation(DA) methods such as ensemble Kalman filter has gained popularity in the Earth sciences. Early attempts, for example in hydrogeology, uses the standard filtering approach to update both parameter and state variables (e.g. Camporese et al. 2015; Zhou et al. 2014) but recent work has focused on reformulating the problem to estimate model parameters only (e.g. Song et al. 2019; Chen et al. 2013). The ensemble or Monte Carlo nature of such methods allows them to produce uncertainty estimates but unlike MCMC, the relatively small number of samples and the assumed multi-variate Gaussianity in the posterior parameter space implies that they can only derive one peak for each parameter value and its spread. Ensemble smoothers or ensemble Kalman inversion (EKI) are also sometimes seen as an alternative to classical inversion solvers, which require the derivation of sensitivity matrices. 
For many large and complex coupled problems, this is not a trivial task while DA methods only require the evaluation of forward model runs.

While classical geosatistical approaches treat the model domain as a continuous random field, recent methods aim to treat the model domain as discrete facies (i.e. zones). Note that these facies may not be geological but can also be "geochemical or hydrological" for modelling purposes (e.g. Sassen et al. 2012; Wainwright et al. 2014). Among them, the level set method (Chan \& Tai 2004) represents boundaries between zones or facies by using the zero contour (the "level set") of a scalar function. One of its earliest applications is to a simplistic electrical resistivity imaging problem (Chung et al. 2005). Notable applications of level set parametrization of the model parameter field includes a Bayesian inversion method of pump test data that is extensible to geophysical data (Cardiff \& Kitanidis 2009) and an ensemble data assimilation framework for pump test and tracer data at the U.S. Hanford site (Song et al. 2019). In addition, it has been applied to study stream bed heterogeneity in a hyporheic exchange context (Chen \& Zeng 2015).

Recognizing the advantages and limitations of current methods, we propose an ensemble Kalman inversion combined with level set parametrization for electrical resistivity imaging. Its Bayesian and Monte Carlo nature provides estimates of model uncertainty at a fraction of the computation cost of MCMC inversion, allowing it to be applied readily to large 3D and time-lapse surveys. Its level set parametrization permits the estimation of spatially varying geostatistical parameters of the resistivity field within the inversion so that it can capture elongated features with different orientations. Its use of Matérn covariance functions allows estimation of spatially variable correlation lengths of the resistivity field. We describe the methodology in the next section and demonstrate its use with a series of synthetic and field hydrogeophysics examples, followed by a discussion and conclusions.

\section{MATERIALS AND METHODS}

\subsection{Bayesian formulation of ERT}

For any given ERT modelling setting under consideration, we denote by $\mathcal{F}: \Sigma \rightarrow \mathcal{V}$ the abstract (forward) operator that maps subsurface electrical conductivity $\sigma(x)$ (i.e. $1 /$ resistivity) into predictions of electrical potential at the electrodes $\mathbf{V}$. Here $\Sigma$ is the space of physically admissible conductivities and $\mathcal{V}$ is the space of all possible geoelectrical measurements. As an example, consider 3D surface ERT with a single (generic) dipole located at $x_{A}$ and $x_{B}$ where electrical current, $I$, is injected. In this case, $\mathcal{F}(\sigma)=\mathbf{V}=\left(V\left(x_{M}\right), V\left(x_{N}\right)\right)$ consists of 
the predictions of electrical potential at the measurement dipole at $x_{M}$ and $x_{N}$. Assuming an isotropic medium, the electrical potential difference between the two points $\Delta V$ can be obtained by solving:

$$
-\nabla \cdot(\sigma \nabla V)=I\left(\delta\left(x-x_{A}\right)-\delta\left(x-x_{B}\right)\right)
$$

with appropriate boundary conditions (e.g. Binley 2015).

Given a set of observations, $d$, collected from measurement electrodes, an ERT problem consists of finding the subsurface conductivity, denoted by $\sigma^{\dagger}(x)$. In the classical (deterministic) ERT setting, an estimate of $\sigma^{\dagger}(x)$ is obtained by minimizing, over the space $\Sigma$, the following functional (e.g. Binley 2015):

$$
\frac{1}{2}\left\|W_{d}(d-\mathcal{F}(\sigma))\right\|^{2}+\frac{\beta}{2}\left\|W_{\sigma} \sigma\right\|^{2},
$$

where $W_{d}$ is a matrix that assigns weights (precision) to the data, $\beta$ is a tuning regularization parameter and $W_{\sigma}$ is often a differential operator that enforces smoothness in a minimizer of (2). Estimating $\sigma$ via minimizing (2), often using the Gauss-Newton method, is usually called smoothness constrained ERT. Note that the $\frac{1}{2}$ constant is usually not included in the geophysics literature, but it is included in order to be consistent with the maximum-aposteriori (MAP) estimate described later.

Rather than computing a single estimate of the true $\sigma^{\dagger}(x)$, in the Bayesian framework (Kaipio \& Somersalo 2005; Stuart 2010) we aim to compute the probability distribution of $\sigma(x)$ conditioned on the observed geoelectrical measurements, $d$. We assume $\sigma(x)$ is a random function with a prior distribution, $\mathbb{P}(\sigma)$, that encapsulates our knowledge of the conductivity before the measurements are collected. Observations/data $d$ are treated as a realization of a random vector. Once measurements become available, the goal of the Bayesian setting is to approximate the so called posterior distribution $\mathbb{P}(\sigma \mid d)$ which, from Bayes' rule, is given by

$$
\mathbb{P}(\sigma \mid d)=\frac{1}{Z} \mathbb{P}(\sigma) \mathbb{P}(d \mid \sigma)
$$

where $\mathbb{P}(d \mid \sigma)$ is the likelihood (i.e. the probability of $d$ given a realization $\sigma$ ), and $Z$ is a normalization factor such that $\mathbb{P}(\sigma \mid d)$ integrates to one. In order to define the likelihood, we use standard assumptions in which the unknown, $\sigma(x)$, and the data, $d$, are related via

$$
d=\mathcal{F}(\sigma)+\eta
$$

where $\eta$ is (unknown) measurement error. Following standard practice, we assume the distribution of $\eta$ is centered Gaussian: $\mathbb{P}_{\eta}(\eta)=N(0, \Xi)$, where here $N(m, C)$ denotes a Gaussian measure with mean $m$ and covariance $C$. From this assumption and (4), and following (Kaipio 
\& Somersalo 2005; Stuart 2010), (3) can be written as

$$
\mathbb{P}(\sigma \mid d)=\frac{1}{Z} \mathbb{P}(\sigma) \exp \left[-\frac{1}{2}\left\|\Xi^{-1 / 2}(d-\mathcal{F}(\sigma))\right\|^{2}\right]
$$

where here $Z$ denotes a (new) normalization factor that we write explicitly as follows:

$$
Z \equiv \int_{\Sigma} \mathbb{P}(\sigma) \exp \left[-\frac{1}{2}\left\|\Xi^{-1 / 2}(d-\mathcal{F}(\sigma))\right\|^{2}\right] d \sigma
$$

In order to fully determine the posterior $\mathbb{P}(\sigma \mid d)$ from (5), we face two substantial challenges. The first one is to design a good prior $\mathbb{P}(\sigma)$ that not only reflects our prior knowledge of $\sigma$, but is also capable of extracting key features of the true $\sigma^{\dagger}$. The second challenge is to compute $Z$. Indeed, if $\mathbb{P}(\sigma)$ is specified and $Z$ is known, then expression (5) fully determines the posterior that can be, in turn, used to compute point estimates (e.g. mean) as well as measures of uncertainty (i.e. variance and credible intervals) for $\sigma(x)$.

Unfortunately, $Z$ cannot be computed analytically for ERT because the underlying forward operator $\mathcal{F}(\sigma)$ is nonlinear. The numerical approximation of (6), on the other hand, is computationally unfeasible. More specifically, after discretizing $\sigma(x)$ on a computational domain, the sought unknowns become a vector with the values of conductivity at, say $K$, cells/elements of the computational domain for the geoelectrical problem (e.g. eq. (1)). Then (6) becomes an integration on a $K$-dimensional space which, for large-scale 3D settings, can be as large as $10^{6}$. In order to address this limitation, sampling algorithms such as MCMC are often used, since they can produce samples of $\mathbb{P}(\sigma \mid d)$ without the need for knowledge of $Z$. If $J$ posterior samples, $\left\{\sigma^{(j)}(x)\right\}_{j=1}^{J}$, are available from a sampling a method, statistics of $\mathbb{P}(\sigma \mid d)$ can be approximated via Monte Carlo sampling. For example, the posterior mean, $E[\sigma \mid d]$, and posterior variance, $\operatorname{Var}[\sigma \mid d]$, can be approximated by the sample mean and sample variance:

$$
\begin{aligned}
E[\sigma \mid d] & \approx \frac{1}{J} \sum_{j=1}^{J} \sigma^{(j)}(x) . \\
\operatorname{Var}[\sigma \mid d] & \approx \frac{1}{J-1} \sum_{j=1}^{J}\left(\sigma^{(j)}(x)-\bar{\sigma}(x)\right)^{2} .
\end{aligned}
$$

Before we proceed to the discussion on the choice of prior as well as the sampling method to approximate the Bayesian posterior for ERT, we briefly discuss the link between the Bayesian approach and the smoothness constrained optimization defined in terms of (2). To this end, let us define the so-called maximum-a-posteriori (MAP) estimate which is the point estimate of the posterior defined by

$$
\sigma_{M A P} \equiv \max _{\sigma \in \Sigma} \mathbb{P}(\sigma \mid d)
$$


This quantity is often used to estimate the truth $\sigma^{\dagger}(x)$ since the MAP can be interpreted as the most likely estimate. Let us assume first that the prior is Gaussian, say $\mathbb{P}(\sigma)=N\left(0, C_{\sigma}\right)$. Under this assumption, (3) can be written as

$$
\mathbb{P}(\sigma \mid d)=\frac{1}{Z} \exp \left[-\frac{1}{2}\left\|\Xi^{-1 / 2}(d-\mathcal{F}(\sigma))\right\|^{2}\right] \exp \left[-\frac{1}{2}\left\|C_{\sigma}^{-1 / 2} \sigma\right\|^{2}\right]
$$

where $Z$ is a (new) normalizing factor defined, as before, such that the posterior $\mathbb{P}(\sigma \mid d)$ integrates to one. Maximizing the posterior $\mathbb{P}(\sigma \mid d)$ in (10) (i.e. computing the MAP) is equivalent to minimizing

$$
-\log (\mathbb{P}(\sigma \mid d))=\frac{1}{2}\left\|\Xi^{-1 / 2}(d-\mathcal{F}(\sigma))\right\|^{2}+\frac{1}{2}\left\|C_{\sigma}^{-1 / 2} \sigma\right\|^{2}+Z
$$

If $W_{d}=\Xi^{-1 / 2}$ and $\beta^{2} W_{\sigma}=C_{\sigma}^{-1 / 2}$, then computing the MAP estimator is equivalent to minimizing (2) which, in turn, leads to the smoothness constrained solution (recall $Z$ is a constant hence fixed through the optimization). For most common choices of prior covariance, $C_{\sigma}^{-1 / 2}$, is indeed a differential operator that enforces smoothness on $\sigma$. This means that a selection of Gaussian prior in the Bayesian approach is not the best choice when our main concern is to characterize discontinuous conductivities via ERT.

The connection between smoothness constrained inversion and the MAP estimator can be further generalized to non-Gaussian priors by considering an appropriate penalty term, $\frac{\beta}{2}\left\|W_{\sigma} \sigma\right\|^{2}$, in (2). However, the advantage of the Bayesian approach over smoothness constrained is that, with the former we have the full posterior $\mathbb{P}(\sigma \mid d)$ that we can use to quantify uncertainty in the estimates of $\sigma(x)$.

\subsection{Priors and parameterizations}

In addition to quantifying uncertainty in the estimates that we produce, a key requirement for our ERT framework is to be able to capture sharp interfaces between zones of different conductivity. In the Bayesian setting this can be done either by (i) a careful selection of priors $\mathbb{P}(\sigma)$ that produces realizations of conductivity fields with discontinuities or (ii) a parameterization of the conductivity field which incorporates discontinuous (or piecewise continuous) features. As discussed at the end of the preceding section, a Gaussian prior will enforce smoothness via the prior covariance. Hence, Gaussian priors are not suitable for characterizing discontinuous (non-smooth) fields. Other priors that can be used for this purpose are the so-called edgepreserving (i.e. Total Variation, Besov and Cauchy) priors (Arridge et al. 2019) commonly used in image processing. However, unlike Gaussian priors, characterizing (e.g. sampling from) these edge-preserving distributions, can be computationally complex. For this reason we fol- 
low the second approach which consists of an adequate parameterization of $\sigma=\mathcal{P}(u)$ that we construct so that (i) it allows us to characterize multiple (unknown) zones with different conductivity and (ii) the new level set parameter $u(x)$ can be easily characterized under the prior. In subsection 2.3 we revisit the Bayesian ERT formulation from subsection 2.1 in terms of the new parameter $u(x)$.

For the sake of exposition we consider a 3-zone example where the conductivity $\sigma(x)$ is defined for $x \in \mathbb{R}^{d}$ ( $d=2$ or $d=3$ for $2 \mathrm{D}$ and $3 \mathrm{D}$ problems, respectively), but the approach can be used for more zones. Constructing the parameterization involves two steps.

\subsubsection{Level-set parameterization}

First, we parameterize our unknown conductivity $\sigma(x)$ in terms of four (also unknown) functions $\xi_{1}(x), \xi_{2}(x), \xi_{3}(x)$, and $\xi_{4}(x)$, via the following expression

$$
\sigma(x)=\mathcal{P}^{L S}\left(\xi_{1}, \xi_{2}, \xi_{3}, \xi_{4}\right) \equiv \begin{cases}\exp \left(\xi_{1}(x)\right), & \xi_{4}(x) \leq \alpha_{1} \\ \exp \left(\xi_{2}(x)\right), & \alpha_{1}<\xi_{4}(x) \leq \alpha_{2} \\ \exp \left(\xi_{3}(x)\right), & \xi_{4}(x)>\alpha_{2}\end{cases}
$$

where $\alpha_{1}$ and $\alpha_{2}$ are user defined parameters. From (12) it follows that $\xi_{4}(x)$, to which we refer as the level-set function, defines three regions

$$
\Omega_{1}=\left\{x: \xi_{4}(x) \leq \alpha_{1}\right\}, \quad \Omega_{2}=\left\{x: \alpha_{1}<\xi_{4}(x) \leq \alpha_{2}\right\}, \quad \Omega_{3}=\left\{x: \xi_{4}(x)>\alpha_{2}\right\}
$$

on which $\sigma(x)$ takes the values given by $\exp \left(\xi_{1}(x)\right), \exp \left(\xi_{2}(x)\right)$ and $\exp \left(\xi_{3}(x)\right)$ respectively. Note that the $\alpha_{1}$-level-set of $\xi_{4}(x)$ (i.e. $\left.\left\{x: \xi_{4}(x)=\alpha_{1}\right\}\right)$ gives the interface between $\Omega_{1}$ and $\Omega_{2}$. Similarly, the $\alpha_{2}$-level-set of $\xi_{4}(x)$ describes the interface between $\Omega_{2}$ and $\Omega_{3}$.

Instead of estimating $\sigma(x)$ we could now reformulate the inverse problem in terms of identifying the functions $\left\{\xi_{\iota}(x)\right\}_{\iota=1}^{4}$. Solving this new problem will amount to estimating the shape of the three zones (via $\xi_{4}(x)$ ) of different conductivity, together with the possibly heterogeneous conductivities within each zone. Before we tackle this new inverse problem, we need to further parameterize $\left\{\xi_{\iota}(x)\right\}_{\iota=1}^{4}$.

\subsubsection{Whittle-Matérn parameterization}

We introduce a second step in the parameterization of $\sigma(x)$ which involves the characterization of the spatial variability of the unknown function $\left\{\xi_{\iota}(x)\right\}_{\iota=1}^{4}$ that we introduced in (12). Our main modelling assumption is that, under the prior, all these functions are Gaussian random 

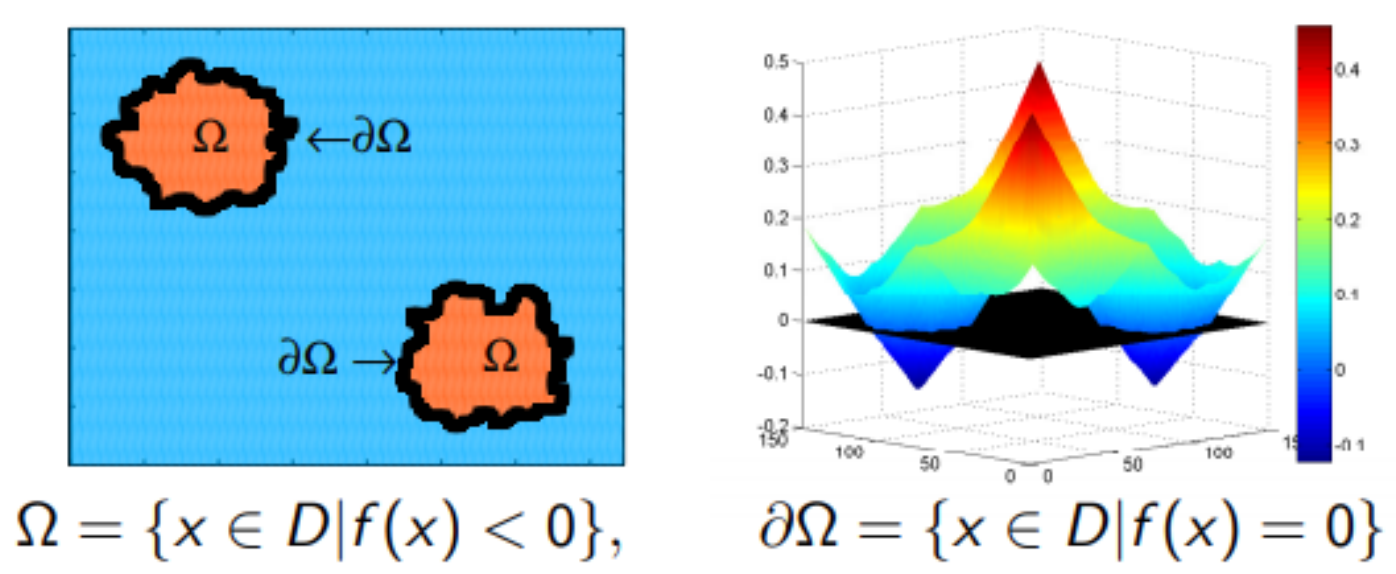

Figure 1. An illustration of the level set methods to delineate sharp interfaces. Using a higher dimension function allows much more straightforward description of arbitrary geometries in the original dimension. In this illustration, the two zones can be defined simply as above or below $z=0$ level of the $3 \mathrm{D}$ function.

fields (GRFs) with Matérn covariance defined by

$$
C_{\Theta}\left(x, x^{\prime}\right)=\tau^{2} \frac{1}{2^{\nu-1} \Gamma(\nu)}\left\|x-x^{\prime}\right\|_{\mathbf{L}}^{\nu} K_{\nu}\left(\left\|x-x^{\prime}\right\|_{\mathbf{L}}\right),
$$

where $\nu$ controls smoothness of the GRFs; $\mathbf{L}=\left(L_{1}, \ldots, L_{d}\right)$ is a vector with the intrinsic length-scales on each of the $d$ directions, $\tau$ is an amplitude scale, $K_{\nu}$ is the modified Bessel function of the second kind of order $\nu, \Gamma$ is the gamma function, and

$$
\left\|x-x^{\prime}\right\|_{\mathbf{L}} \equiv \sqrt{\frac{\left(x_{1}-x_{1}^{\prime}\right)^{2}}{L_{1}^{2}}+\cdots+\frac{\left(x_{d}-x_{d}^{\prime}\right)^{2}}{L_{d}^{2}}} .
$$

The (hyper-)parameters of (14) are comprised in a vector $\Theta=(\mathbf{L}, \nu, \tau)$.

Suppose that a generic function $\xi(x)$ is Gaussian random field with mean $\lambda$ and covariance $C_{\Theta}$ (we denote this by $\xi \sim N\left(\lambda, C_{\Theta}\right)$ ). One of the most common approaches to characterize $\xi(x)$ is via its Karhunen-Loeve (KL) expansion given by

$$
\xi(x)=\lambda+\sum_{i=1}^{\infty} \zeta_{\Theta, i}^{1 / 2} v_{\Theta, i}(x) \kappa_{i},
$$

where $\left(\zeta_{\Theta, i}, v_{\Theta, i}(x)\right)$ is the eigenvalue-eigenfunction pair of $C_{\Theta}$ and the $\kappa_{i}$ 's are independent identically distributed (iid) samples from a standard normal (i.e. $\theta_{i} \sim N(0,1)$ ). The KL expansion is quite general and it can be used for a more general class of covariance operators. When $C_{\Theta}$ is given by (14), and the GRF is defined on a domain with simple geometry (i.e. a rectangular domain (Dunlop et al. 2017)), analytical expressions for the eigen-pair $\left(\zeta_{\Theta, i}, v_{\Theta, i}(x)\right)$ may be available. Constructing the eigen-pair for the KL characterization of GRFs is equivalent to 
the Stochastic Partial Differential (SPDE) approach proposed in (Lindgren et al. 2011), and that we adopt for the present work. The SPDE approach consists of expressing a GRF with Matérn covariance as follows

$$
\xi(x)=\mathcal{P}^{W M}(\lambda, \Theta, \omega) \equiv \lambda+\mathcal{W}_{\Theta} \omega(x)
$$

where here $\omega(x)$ is Gaussian white noise at each grid location $x$ and $\Psi=\mathcal{W}_{\Theta} \omega$ is the solution to the fractional Stochastic Partial Differential (SPDE)

$$
\left(\mathbb{I}-\nabla \cdot \operatorname{diag}\left(\mathbf{L}^{2}\right) \nabla\right)^{(\nu+d / 2) / 2} \Psi=\tau^{2} 2^{d} \pi^{d / 2} \frac{\Gamma(\nu+d / 2)}{\Gamma(\nu)}\left[\prod_{i=1}^{d} L_{i}\right]^{1 / 2} \omega
$$

together with appropriate boundary conditions (Roininen et al. 2014). In the previous expression $\mathbb{I}$ denotes the identity operator and $\operatorname{diag}\left(\mathbf{L}^{2}\right)$ is a matrix (either in $2 \mathrm{D}$ or $3 \mathrm{D}$ ) with diagonal equal to $\mathbf{L}^{2}=\left(L_{1}^{2}, \ldots, L_{d}^{2}\right)$.

While the characterization of GRFs via (16)-(17) may seem quite involved, we should note that the operator $\nabla \cdot \operatorname{diag}\left(\mathbf{L}^{2}\right) \nabla$ that appears in (17) is simply the anisotropic version of a Laplacian. Thus, a discretization of $\mathcal{A} \equiv\left(\mathbb{I}-\nabla \cdot \operatorname{diag}\left(\mathbf{L}^{2}\right) \nabla\right)$ can be relatively straightforward using standard Finite Element or Finite Difference methods. Given the discretization of $\mathcal{A}$, the SPDE Approach from Lindgren et al. (2011) provide us with straightforward steps for solving (17) for the case when $(\nu+d / 2)$ is an integer.

We propose to characterize each of the functions $\left\{\xi_{\iota}(x)\right\}_{\iota=1}^{4}$ that we introduced in (12) via the parameterization induced by the SPDE approach. In other words, we assume each of these functions are, under the prior, GRFs that can be written as

$$
\xi_{i}(x)=\mathcal{P}^{W M}\left(\lambda_{i}, \Theta_{i}, \omega_{i}\right) \quad i=1, \ldots, 4
$$

The hyper-parameters $\Theta_{i}=\left(\mathbf{L}_{i}, \nu_{i}, \tau_{i}\right)$ in (18) are crucial since they control the spatial variability of each $\xi_{i}(x)$. The work in Chada et al. (2018) and Dunlop et al. (2017) has shown that, prior hyper-parameters of this kind, must be estimated within the Bayesian setting to enable accurate characterizations of physical properties. In particular, the hyper-parameters, $\Theta_{4}$ for the level-set function $\xi_{4}(x)$ will determine the geometry of each of the regions defined in (13) as well as the smoothness of the interface across these regions.

We combine (18) with (12) to define our parameterization of $\sigma(x)$ :

$$
\sigma(x)=\mathcal{P}^{L S}\left(\xi_{1}, \xi_{2}, \xi_{3}, \xi_{4}\right)=\mathcal{P}^{L S}\left(\mathcal{P}^{W M}\left(\lambda_{1}, \Theta_{1}, \omega_{1}\right), \ldots, \mathcal{P}^{W M}\left(\lambda_{4}, \Theta_{4}, \omega_{4}\right)\right)
$$

which in compact form can be written as

$$
\sigma(x)=\mathcal{P}(u(x)), \quad u(x)=\left(\lambda_{1}, \Theta_{1}, \omega_{1}(x), \ldots, \lambda_{4}, \Theta_{4}, \omega_{4}(x)\right)
$$


Rather than approximating the posterior distribution for the $(\log )$ conductivity $\sigma(x)$, our goal is to compute the posterior on $u(x)$ and then transform back to a distribution on $\sigma(x)$ via $\mathcal{P}$. The framework above gives us a natural choice for the priors $\mathbb{P}\left(\omega_{i}\right)=N(0, \mathbb{I})$ (i.e. Gaussian white noise). Priors on $\lambda_{i}$ and $\Theta_{i}=\left(\mathbf{L}_{i}, \nu_{i}, \tau_{i}\right)$ must now be specified.

\subsection{Ensemble Kalman inversion (EKI) for the parameterized ERT problem}

In this section we apply the Bayesian framework to the new parameter $u(x)$ that we introduce earlier to characterize discontinuous $\sigma(x)$ via the mapping $\sigma=\mathcal{P}(u)$. We re-formulate the Bayesian ERT problem in terms of the push-forward of $\mathbb{P}(u \mid d)$ (i.e. the posterior on $u(x)$ ) under the parameterization map $\mathcal{P}$. In plain words, if we are able to produce samples from $\mathbb{P}(u \mid d)$, say $\{u(j)\}_{j=1}^{J}$, then the corresponding conductivities, $\sigma^{(j)}=\mathcal{P}\left(u^{(j)}\right)$, are samples of the "push-forward" density that we denote by $\mathbb{P}^{y}(\sigma)$. This is analogous to the posterior on $\sigma(x)$ introduce in the subsection 2.1 and comprises the statistical information of $\sigma(x)$ while enforcing the 3-zone assumption encoded in $\mathcal{P}(u)$.

The posterior on $u(x)$ is given, again, by Bayes rule

$$
\mathbb{P}(u \mid d)=\frac{1}{Z} \mathbb{P}(u) \mathbb{P}(d \mid u)
$$

where $\mathbb{P}(u)$ is the prior on $u$ and $\mathbb{P}(d \mid u)$ is the likelihood. We define the composition map $\mathcal{G}(u)=\mathcal{F} \circ \mathcal{P}(u)=\mathcal{F}(\sigma)$ that maps the parameter $u(x)$ into the prediction of the geoelectrical model $\mathcal{F}(\sigma)=\mathbf{V}$. We use the parameterization $\sigma=\mathcal{P}(u)$ as well as the definition of $\mathcal{G}$ in (4) to find

$$
d=\mathcal{F}(\sigma)+\eta=\mathcal{F}(\mathcal{P}(u))+\eta=\mathcal{G}(u)+\eta
$$

Using again our assumption on Gaussian measurement noise $\eta \sim N(0, \Xi)$, we rewrite (21) as

$$
\mathbb{P}(u \mid d)=\frac{1}{Z} \mathbb{P}(u) \exp \left[-\frac{1}{2}\left\|\Xi^{-1 / 2}(y-\mathcal{G}(u))\right\|^{2}\right],
$$

\subsubsection{EKI algorithm}

In order to approximate the posterior $\mathbb{P}(u \mid d)$ in $(22)$, we consider the ensemble Kalman inversion (EKI) algorithm of Iglesias et al. (2018). EKI is based on the tempering scheme which consist of introducing a sequence of $N$ intermediate densities between prior and posterior:

$$
\mathbb{P}_{0}(u) \rightarrow \mathbb{P}_{1}(u) \rightarrow \ldots \mathbb{P}_{N}(u) \rightarrow \mathbb{P}_{N+1}(u)=\mathbb{P}(u \mid d)
$$

Each intermediate density is defined by

$$
\mathbb{P}_{n}(u) \propto \mathbb{P}_{0}(u) \exp \left[-\frac{\phi_{n}}{2}\left\|\Xi^{-1 / 2}(d-\mathcal{G}(u))\right\|^{2}\right],
$$


where $\left\{\phi_{n}\right\}_{n=1}^{N}$ are tempering parameters that satisfy:

$$
\phi_{0} \equiv 0<\phi_{1}<\phi_{2}<\cdots<\phi_{N}<\phi_{N+1} \equiv 1 \text {. }
$$

Note that $n=0$ and $n=N+1$ in $(23)$ corresponds to the prior, $\mathbb{P}_{0}(u)$, and posterior, $\mathbb{P}_{N+1}(u)=\mathbb{P}(u \mid d)$, respectively. The following recursive formula can be obtained from (23):

$$
\mathbb{P}_{n+1}(u) \propto \mathbb{P}_{n}(u) \exp \left[-\frac{1}{2}\left\|\left(\alpha_{n} \Xi\right)^{-1 / 2}(d-\mathcal{G}(u))\right\|^{2}\right]
$$

where

$$
\alpha_{n}^{-1}=\phi_{n+1}-\phi_{n}
$$

From (24) it follows that

$$
\sum_{n=0}^{N} \alpha_{n}^{-1}=\phi_{N+1}-\phi_{0}=1
$$

EKI is an iterative algorithm that, at each of the $n$-th iteration, produces samples from a Gaussian approximation of $\mathbb{P}_{n}(u)$. The algorithm is initialized with an ensemble of inputs $\left\{u_{0}^{(j)}\right\}_{j=1}^{J}$ drawn from the prior $\mathbb{P}(u)$, measurements $d$ and error covariance $\Xi$. Set $n=0$ and $\theta_{-1}=0$

At each iteration $n$,

(1) Compute $\mathcal{G}_{n}^{(j)}=\mathcal{G}\left(u_{n}^{(j)}\right), \quad j \in\{1, \ldots, J\}$ by running the forward model.

(2) Compute $\alpha_{n}$ via

$$
\alpha_{n}^{-1} \equiv \min \left\{\left[\frac{1}{M} \frac{1}{J} \sum_{j=1}^{J}\left\|\Xi^{-1 / 2}\left(d-\mathcal{G}_{n}^{(j)}\right)\right\|^{2}\right]^{-1}, 1-\theta_{n-1}\right\}
$$

where $M$ is the number of measurements (i.e. length of vector $d$ ) and set $\theta_{n} \leftarrow \theta_{n-1}+$ $\alpha_{n}^{-1}$. The choice of regularization parameter $\alpha_{n}$ is crucial to inversion performance; here we follow the approach proposed recently in Iglesias \& Yang (2020).

(3) Update each particle (a realization of the ensemble) according to:

$$
u_{n+1}^{(j)}=u_{n}^{(j)}+\mathcal{C}_{n}^{u \mathcal{G}}\left(\mathcal{C}_{n}^{\mathcal{G G}}+\alpha_{n} \Xi\right)^{-1}\left(d+\sqrt{\alpha_{n}} \eta_{n}^{(j)}-\mathcal{G}\left(u_{n}^{(j)}\right)\right), \quad j \in\{1, \ldots, J\}
$$

where $\eta_{n}^{(j)} \sim N(0, \Xi)$ is artificial noise that we generate with the same assumed statistics of the measurement error. In eq. (29), $C_{n}^{u \mathcal{G}}$ and $C_{n}^{\mathcal{G G}}$ are model-data covariance and data auto-covariance matrices respectively, which are formed empirically based on the evaluation 
of the forward models $\left\{\mathcal{G}\left(u_{n}^{(j)}\right)\right\}_{j=1}^{J}$. Specifically, they are given by:

$$
\begin{aligned}
C_{n}^{\mathcal{G}} & \equiv \frac{1}{J-1} \sum_{j=1}^{J}\left(\mathcal{G}\left(u_{n}^{(j)}\right)-\overline{\mathcal{G}}_{n}\right) \otimes\left(\mathcal{G}\left(u_{n}^{(j)}\right)-\overline{\mathcal{G}}_{n}\right) \\
C_{n}^{u \mathcal{G}} & \equiv \frac{1}{J-1} \sum_{j=1}^{J}\left(u_{n}^{(j)}-\bar{u}_{n}\right) \otimes\left(\mathcal{G}\left(u_{n}^{(j)}\right)-\overline{\mathcal{G}}_{n}\right)
\end{aligned}
$$

with $\bar{u}_{n} \equiv \frac{1}{J} \sum_{j=1}^{J} u_{n}^{(j)}$ and $\overline{\mathcal{G}}_{n} \equiv \frac{1}{J} \sum_{j=1}^{J} \mathcal{G}_{n}^{(j)}$.

(4) Set $n \leftarrow n+1$

The iterative loop continues until convergence which is controlled by the criterion in (27), which is equivalent to while $\theta_{n-1}<1$ is not true. At convergence, we yield $\left\{u_{n+1}^{(j)}\right\}_{j=1}^{J}$ as an ensemble approximation of the posterior.

\subsection{Summary of Methods}

To summarize the above, the key point of our method is that we do not directly invert for a distribution of conductivity fields. Rather, we invert for parameters $u$ and use a mapping $\mathcal{P}$ to convert them to conductivity fields. The level set function is a good choice for $\mathcal{P}$ to handle sharp boundaries but we want to parametrize it using hyper-parmeters such as length scales and smoothness. So for each of the level set functions, we rewrite them based on Matérn covariance functions and the SPDE approach (16-18). The end results is that we can parameterize the level set functions $\xi_{i}(x)$ and thus the conductivity fields $\sigma(x)$ through the Whittle-Matérn hyper-parameters for each of the $i$-th zone $\Theta_{i}=\left(\mathbf{L}_{i}, \nu_{i}, \tau_{i}\right)$ via eqn.(18). Recall that $\mathbf{L}_{i}, \nu_{i}$, and $\tau_{i}$ are the controls the length scale, smoothness, and amplitude of the level set functions respectively, alongside the mean conductivity of each zone $\lambda_{i}$ and the Gaussian white noise at each grid location $w_{i}(x)$. Prior conductivity fields are generated by specifying $\lambda_{i}$ and $\Theta_{i}$. For examples of prior conductivity fields, refer to Fig. 4.

The parameters are updated by EKI. The general concept is to update an ensemble of model realizations using ensemble Kalman filter-like updates. By computing the data misfit between the ensemble (obtained by running the forward model for each realization) and the observed, the mean of the ensemble is iteratively driven to the solution of the inverse problem. At each iteration, the regularization parameter $\alpha_{n}$ is updated. It effectively allows more regularization when the data misfit is large, while letting it decreases gradually as the ensemble evolves closer to the solution.

To estimate a conductivity field assuming there is no within-zone heterogeneity, we update $u(x)=\left(\lambda_{i}, \Theta_{i}, w_{i}(x)\right)$ via EKI. $\lambda_{i}$ is the homogeneous conductivity value for each zone. Note 
that although $\lambda$ and $\Theta$ are scalars, we are estimating their distributions (and hence their means and variances).

It is noteworthy that the flexibility of EKI means we may omit certain hyper-parameters or include more if needed. In the deep Earth seismic EKI work of Muir \& Tsai (2020), they are confident about their priors (i.e. the hyper-parameters they set) and the assumed zonal seismic slownesses so these parameters are not updated. In one of their examples, they added a hyper-parameter to account for the potential presence of a fault, which allows them to recover the fault geometry clearly. Since our examples also estimate the length scales, we call our approach "multi-scale", or "hierarchical EKI (Chada et al. 2018)". Although EKI is a Bayesian method, Muir \& Tsai (2020) used it pragmatically as a fast and flexible optimizer to replace the Jacobian and they did not report any uncertainty estimates.

Estimating a conductivity field with within-zone heterogeneity can be achieved by also estimating the spatially varying length scales of within-zone heterogeneity at each direction and at each grid cell. We demonstrate this in one of our synthetic examples.

\subsection{Implementation notes}

For the ERT forward modelling, we use a grid that extends laterally several times the dimension of the ERT imaging area to simulate an infinite earth in field studies. For convenience, here we discretize the parameter grid used for inversion is a grid consists of squares/cubes covering the entirety of the imaging area. For the field hillslope example (section 3.2.1), the parameter grid is distorted to quadrilaterals based on surface elevations. At each iteration, the parameter grid is interpolated to the forward modelling grid to obtain simulated ERT data. In all the examples, the number of realizations used for each iteration is set to 300 . For this paper, we implement the EKI method in $\operatorname{MATLAB}(R)$ and its statistics and machine learning toolbox as well as its built-in parallel tool. However, it can be implemented rather straightforwardly in other scripting languages.

Priors are given in log space and are assumed a uniform distribution. One of the zone is assigned to be the background zones such that its mean value is assigned for cells that are outside the parameter estimation grid.

Putting all of the above together, our approach can be summarized as a flowchart in Fig. 2. The unknown parameters are level set parameters and they are converted to conductivity fields at the beginning of each iteration before being run through the ERT forward code to obtain simulated ERT data.

In all the examples, we compare the inversion results from EKI to smoothness-constrained 


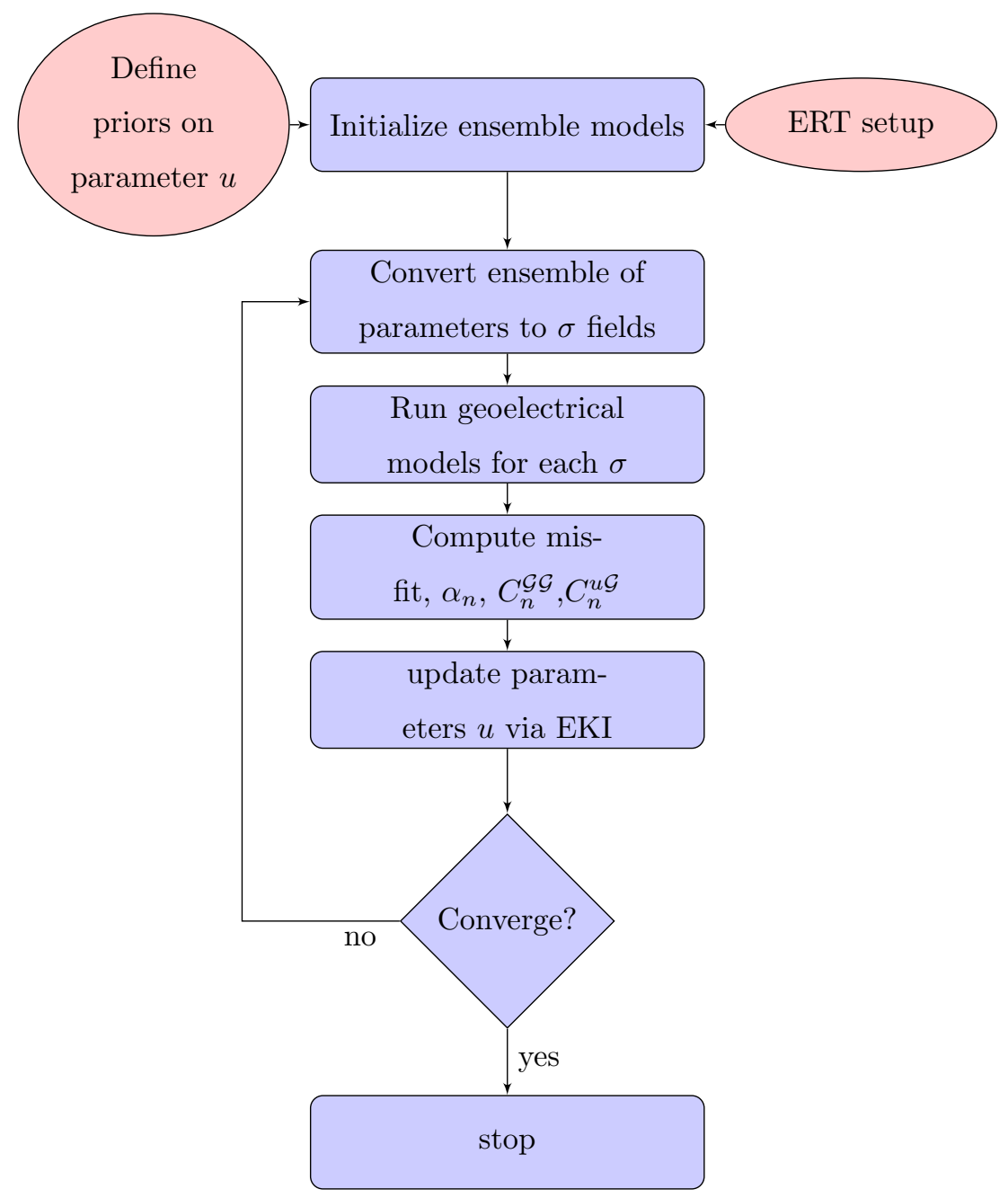

Figure 2. Overview of the EKI method

inversion. The parameter grid used here is the same as the forward modelling grid. R2 and E4D are used for inversion of 2D and 3D data, respectively.

\section{EXAMPLE APPLICATIONS}

In this section, we report EKI inversion results from a series of synthetic and field examples. In these examples, $2 \%$ Gaussian noise is added to the data and a $2 \%$ data errors are assumed in the inversions. For 2D modelling, we use R2 (http://http://www.es.lancs.ac.uk/people/ amb/Freeware/R2/R2.htm) for forward modelling because of its ease to setup the problem, particularly with the help of the the ResIPy interface (Blanchy et al. 2020). For 3D modelling, we use E4D (Johnson et al. 2010) as the forward solver because of its efficient parallel capabilities. For each problem, either a rectangular grid is used or a triangular(2D)/tetrahedron(3D) mesh is generated using tetgen (Si 2015). 


\subsection{Synthetic examples}

\subsubsection{D cross-borehole survey}

In this example, two boreholes, $16 \mathrm{~m}$ apart are installed for cross-borehole ERT imaging (Fig. 3). Along each borehole, 16 regularly separated electrodes are installed. In total, 204 ERT measurements (i.e. quadrupoles) are collected. The forward modelling grid includes 6336 rectangular cells while the parameter grid for inversion consists of 64 regularly spaced square cells in each direction (4096 total), spanning $x=[-8,8] \mathrm{m}$ and $z=[-8,8] \mathrm{m}$. The background resistivity is $100 \Omega \mathrm{m}$ and that of the inclusion is $1 \Omega \mathrm{m}$. The prior resistivity ranges for zone 1 (background) and 2 are $[75,200] \Omega \mathrm{m}$ and $[0.1,30] \Omega \mathrm{m}$ respectively.

The resistivity map obtained from commonly used smoothness constrained inversion is extremely smooth (Fig. 3a) and the resistivity range (note the range of values) is very small. Users familiar with smoothness constrained inversion can appreciate this image may contain a sharp target but it is difficult to communicate this to non-geophysicist. The mean resistivity estimates from EKI (Fig. 3b), in contrast, recovers the two zones very well(with the exception of the corners of the target) and obtains the zonal resistivity value perfectly. The estimated target appears to be shifted to the left slightly from the true rectangular interface-this is due to a rather coarse parameter grid being used. In contrast, the smoothness constrained inversion returns a smoothed images with variations of resistivity values across the image. It also does not give a correct estimate of the resistivity values in neither the background region nor the inclusion.

A helpful feature from EKI is the ability to obtain zonal probability maps of across the estimated resistivity models. Here, since a 2-zone formulation is used, each cell belongs to either the background or the inclusion zone. As can be seen in Fig. 3c, the probability map for zone 1 is highly variable at the first iteration and the values are all between 0.9 to 1.0 , reflecting our assumption of a background region. The posterior map (Fig. 3d), however, shows very high zone 1 probability in most of the domain, very low probability at the center of the inclusion, and a probability of between 0.4 and 0.7 around the interface of the two zones.

\subsubsection{D surface survey}

In this example, a surface ERT line is deployed to image a layered with system with a vertical fault (The true model is shown in Fig. 4a). The layers can be conceptualized as a bedrock overlaid by a less resistive topsoil. 25 regularly separated electrodes ( $2 \mathrm{~m}$ spacing) are installed. In total, 117 dipole-dipole measurements are simulated. The forward modelling grid includes 
(a)

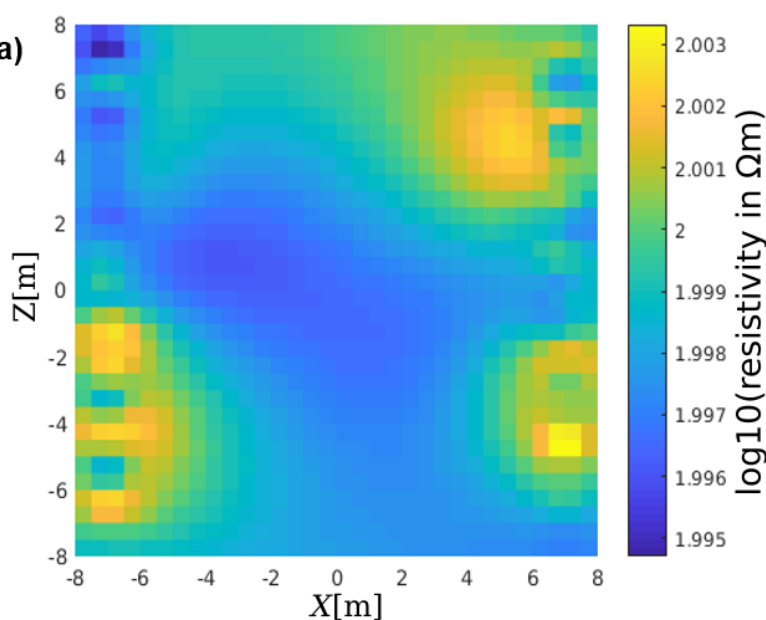

(c)

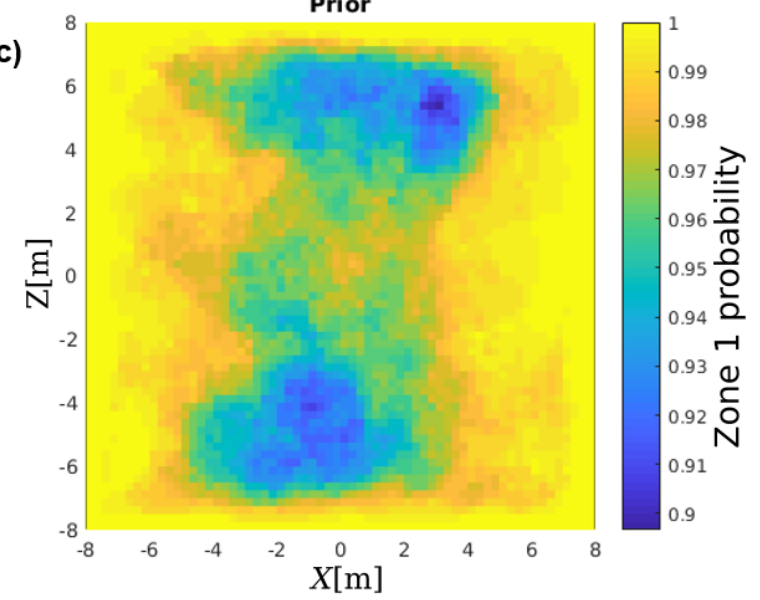

Posterior (iter $=11$ )

(b)

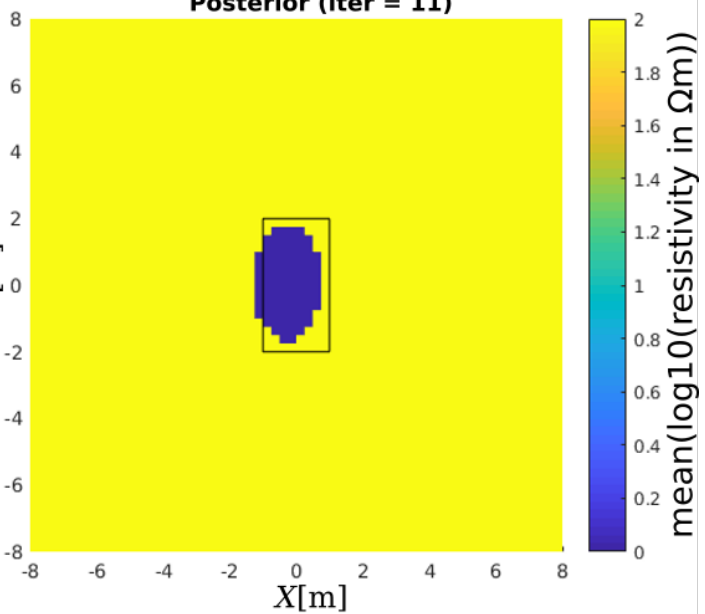

Posterior (iter $=11)$

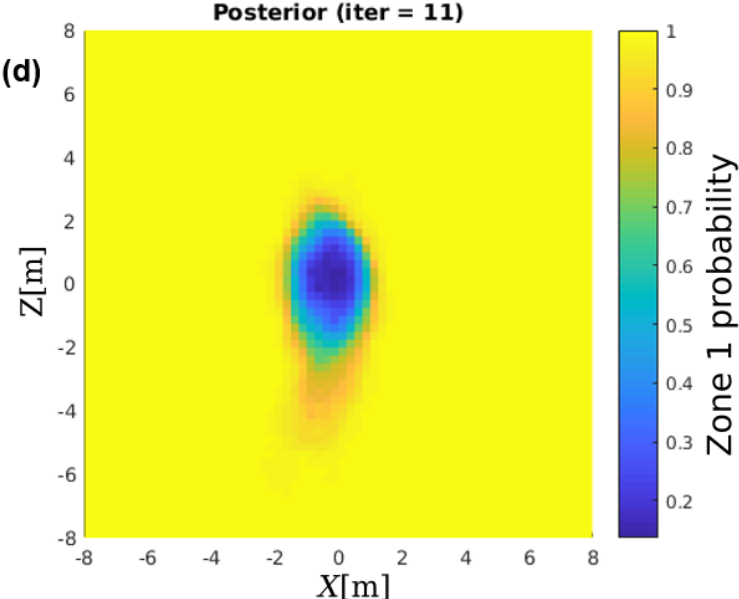

Figure 3. ERT inversion results of the $2 \mathrm{D}$ cross-borehole example: (a)The resistivity model estimated by smoothness-constrained inversion. Notice the smaller resistivity range than the estimates from EKI. (b) The mean resistivity model estimated by EKI, where the rectangular bounding box shows the true location of the $1 \Omega$ m inclusion (c) The prior estimated probability of zone 1 (i.e. the background) (d) The posterior estimated probability of zone 1 . In each sub-figure, the true boundary of the two zones are marked by a red line.

10752 rectangular cells while the parameter grid for inversion consists of 25600 square cells spanning $x=[-24,56] \mathrm{m}$ and $z=[-20,10] \mathrm{m}$, with 320 and 80 regularly spaced cells in the $X$ and $Z$ directions respectively. The background resistivity (zone 1) is $2500 \Omega \mathrm{m}$ and that of the topsoil (zone 2) is $250 \Omega \mathrm{m}$. Fig. 4) also shows a few selected realizations of the prior resistivity fields. They are generated by the level set functions given prior ranges of length scales and resistivity values of the two materials.

Fig. 5 shows the results from a smoothness constrained inversion and a L1 (or blocky) inversion (Loke et al. 2003), respectively. The smoothness constrained inversion shows a more 

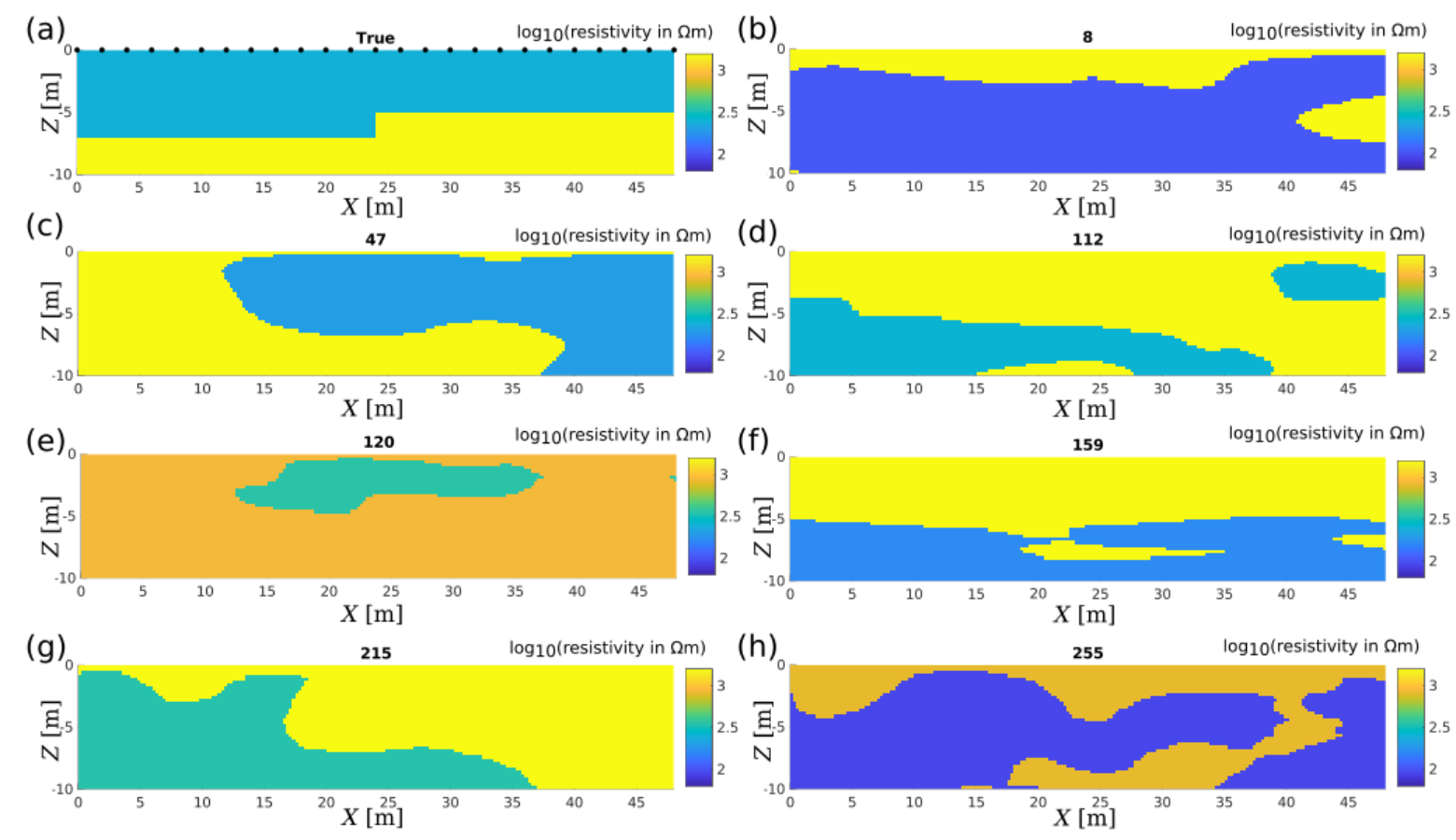

Figure 4. (a) True model for the 2D surface example, which comprises of two layers and a vertical fault. The black circles denotes the electrode locations. (b-h)Example realizations of prior resistivity field and the corresponding realization number.

resistive region at depth greater than $5 \mathrm{~m}$ and the resistivity variation is gradual and smooth. For the L1 inversion, a flat topsoil layer is recovered, while the bedrock to the right of the fault is found (mistakenly) to be less resistive than that to its left. In both inversions, the resistivity value of the bedrock is underestimated.

In this example, EKI with both 2 zone and 3 zone formulations are compared. In layered systems such as this one (i.e. a laterally extended topsoil with different electrical signatures to the rest of the domain), there may be issues in using a 2 zone formulation since the apparent resistivity of most measurements is close to neither zones. Therefore, a formulation with a third zone with a resistivity ranges that is lower than zone 1 and higher than zone 2 is also tested. In the 2 zone example, the prior resistivity of zone 1 (background) and zone 2 are $[2000,3000] \Omega \mathrm{m}$ and $[200,300] \Omega \mathrm{m}$ respectively. Fig. 5 shows some example realizations of prior resistivity fields.

The results for EKI (2 zone) is reported in (Fig. 5c,e,g). It gives a mean estimates of background/bedrock(zone 1) and topsoil (zone 2) resistivity of $2362 \Omega \mathrm{m}$ and $243 \Omega \mathrm{m}$ respectively, which are very close to the true values $(2500$ and $250 \Omega \mathrm{m})$. It also captures the resistivity structure very well-although the recovered fault structure does not appear to be perfectly vertical, the estimate zone boundaries and resistivity values are close to the true ones. As 
shown by Muir \& Tsai (2020), the EKI can be formulated so that fault parameters (e.g. location and dip angle) are estimated explicitly and return more explicit fault geometry (see also discussions in Section 2.4 and 4.3). The variance maps shows the uncertainty (both in terms of variance and coefficient of variation $(\mathrm{CV})$ ) is the highest around the interface between the two zones, which is helpful for interpretation and for uncertainty propagation to subsequent analysis steps. The uncertainty is also lower for the topsoil, partly because it is a lower resistivity layer, but also because of its proximity to the surface electrodes. It is also noteworthy that the left half of the image is subject to higher uncertainty . Recall that the fault system is set up so that the topsoil to the left is $2 \mathrm{~m}$ thicker than that to the right. This observation highlights the attenuation effects of ERT signals due to topsoil thickness.

In the 3 zone formulation (Fig. $5 \mathrm{~d}, \mathrm{f}, \mathrm{h}$ ), the prior resistivity ranges of zone 1 , zone 2 (background) and zone 3 are $[2000,3000] \Omega \mathrm{m},[700,900] \Omega \mathrm{m}$ and $[200,300] \Omega \mathrm{m}$ respectively. The results from EKI ( 2 zone) and EKI ( 3 zones) share many common features. EKI (2 zone) gives a mean estimates of bedrock, background and topsoil resistivity of $2372 \Omega \mathrm{m}, 798 \Omega \mathrm{m}$ and $242 \Omega \mathrm{m}$ respectively, which are very close to the true values and the background value is almost identical to the geometric mean of the other zones. However, the mean estimates designate the cells around the interface between the two zones the moderate background zones. This can be helpful in practice when propagating uncertainty because if the contrast between the two zones are too high, a wrong estimation of zone membership may have serious effects. The variance and $\mathrm{CV}$ maps in the 3 zone formulation also show bands of high uncertainty near the 2 interfaces of the estimated zones.

We repeated our analysis with wider parameter ranges and obtained very similar results. In all the examples, convergence was achieved between 10-12 iterations. The computation was performed on an Intel i7 laptop (quad core) laptop and the time required for the entire inversion is less than 2 hours. The $\mathrm{R} 2$ run for the same problem takes less than a minute on the same machine. Finally, we compare our results with a smoothness-constrained inversion (Fig. $5 \mathrm{~g}$ ), which shows a very smooth interface. Similar to EKI, a $1 \mathrm{~m} \times 1 \mathrm{~m}$ parameter grid was used.

We also considered the performance of EKI to estimate a rather heterogeneous resistivity field. The true field (Fig. 6a) has the same zone boundaries but there are within-zone heterogeneities. The EKI algorithm is set to allow within-zone variations in resistivity values. The EKI algorithm recover the two zones in the true field (Fig. 6b-d), with the exception of a few misidentified small pockets in the top layer. Again, the uncertainty is found to be higher at 
(a)
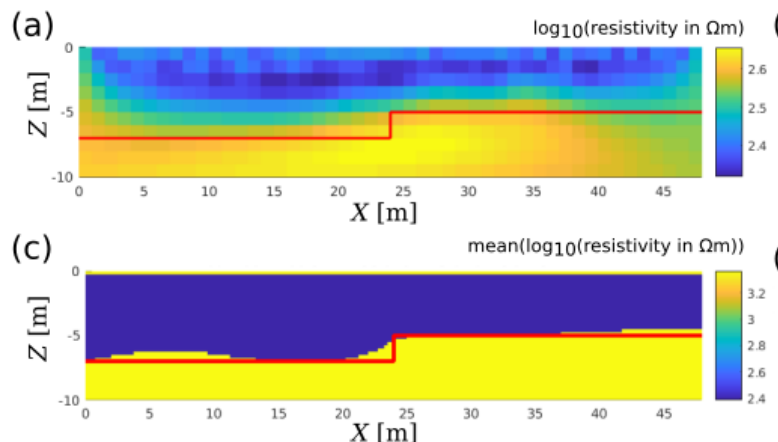

(e)
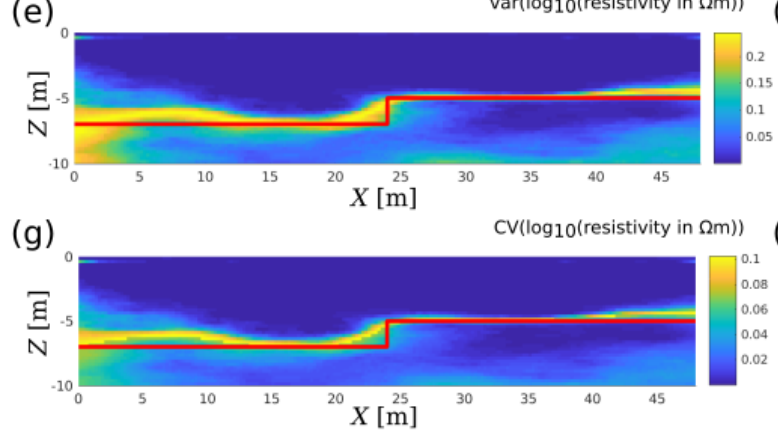

(b)
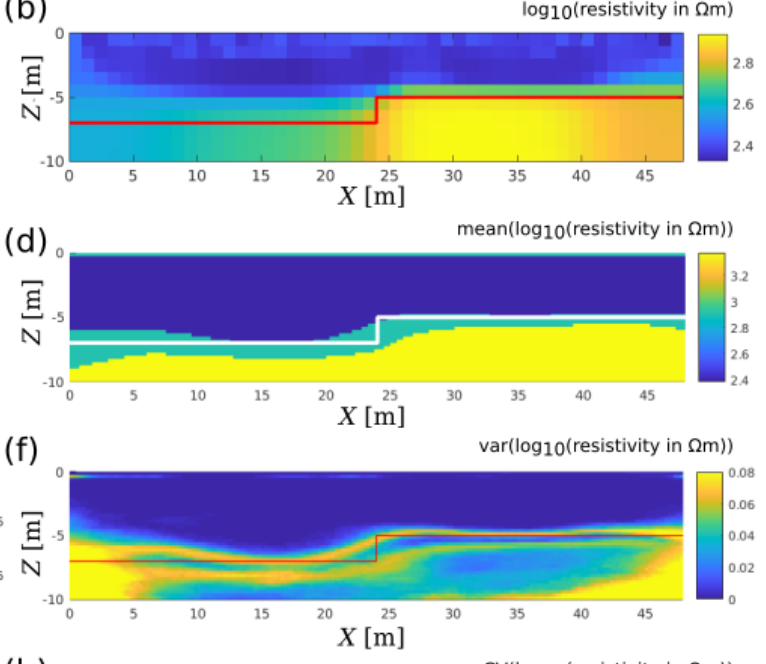

(h)

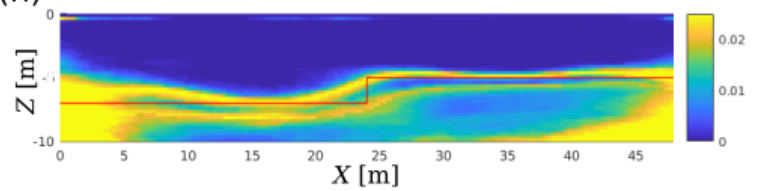

Figure 5. ERT inversion results of the 2D fault example using a 2-zone formulation: (a) The mean resistivity model estimated by EKI (b) The posterior estimated variance. (c) The posterior estimated coefficient of variation (d-f) shows the above by repeating EKI by assuming a 3 -zone formulation.(g)The resistivity model estimated by smoothness-constrained inversion. In each sub-figure, the true boundary of the two zones are marked by a red line. The boundary of the two zones is marked with a red line.

the estimated interfaces between zones. The computation cost for EKI here is comparable to that in Fig. 5.

\subsubsection{D complex structure}

In this $3 \mathrm{D}$ example, the true model is a cylinder of $2 \mathrm{~m}$ radius and $8 \mathrm{~m}$ in height, which mimics structures such as an abandoned well or mine shaft that is backfilled with sediments (Fig. 7). Three surface ERT lines ( $5 \mathrm{~m}$ separation), consisting of 16 electrodes (1 m separation), are used for data collection. The background resistivity is $200 \Omega \mathrm{m}$ and that of the inclusion is $10 \Omega \mathrm{m}$. The ERT grid consists of 62002 tetrahedron cells. For EKI, we set the initial guess vertical length scale hyperparameters $(\mathbf{L})$ to be three times larger than the horizontal ones than to speed up convergence, but note that all of them are updated by EKI. The computation was performed on a DELL PowerEdge cluster.

The smoothness constrained inversion recovers a rather smoothed structure of which the interface between the two zones are not clearly shown. The cylinder appears as a conductive 

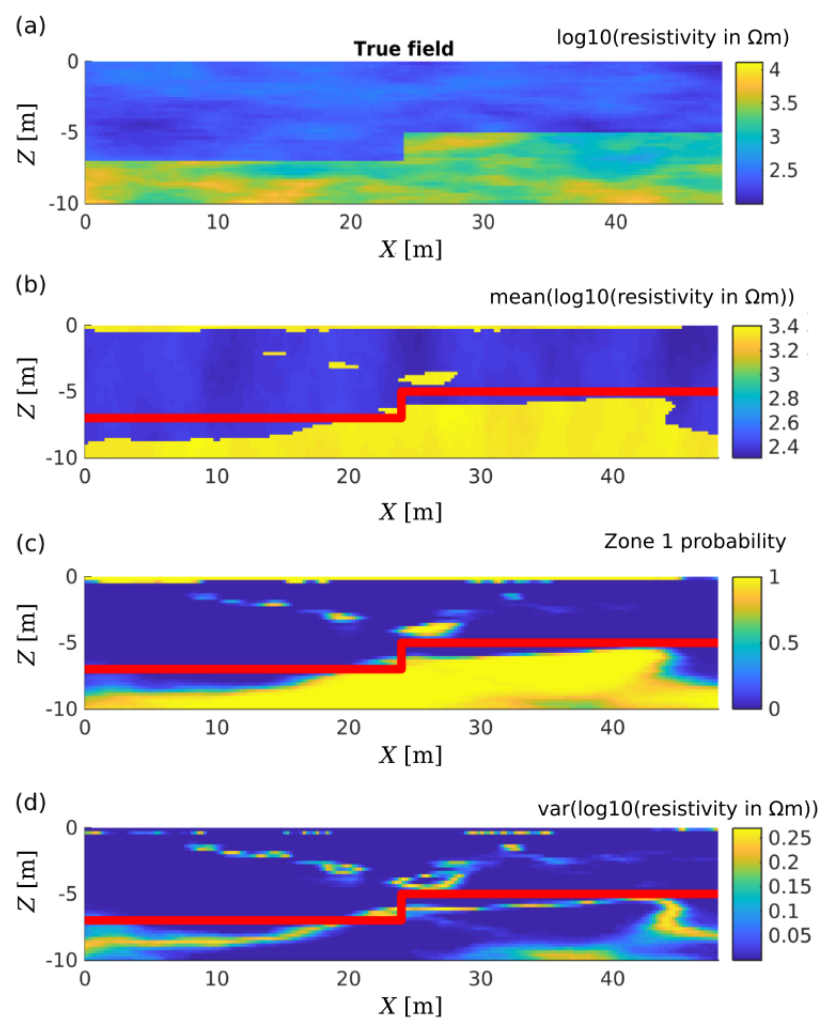

Figure 6. ERT inversion results of the $2 \mathrm{D}$ fault example with heterogeneity within each zone: (a) The true resistivity field (b) The mean resistivity model estimated by EKI (c) The posterior estimated probability of zone 1 (i.e. the background zone) (d)The posterior variance estimated by EKI. In each sub-figure, the true boundary of the two zones are marked by a red line. The boundary of the two zones is marked with a red line.

blob near the surface, making it difficult to interpret if the true resistivity model were not known.

In contrast, the extent of the inclusion is very well recovered by EKI. The mean estimated resistivity for each zone is $200 \Omega \mathrm{m}$ and $10 \Omega \mathrm{m}$, which are very close to their true values. The variance maps shows the confidence of facies/zone estimation and allows propagation of uncertainty for subsequent analysis. The evolution from prior to posterior variance maps shows a reduction in uncertainty by conditional the potential resistivity models with data. The posterior variance is higher near the interface between the two zones. Similarly, the Zone 2 probability allows intuitive visualisation of zone membership and its uncertainty. 

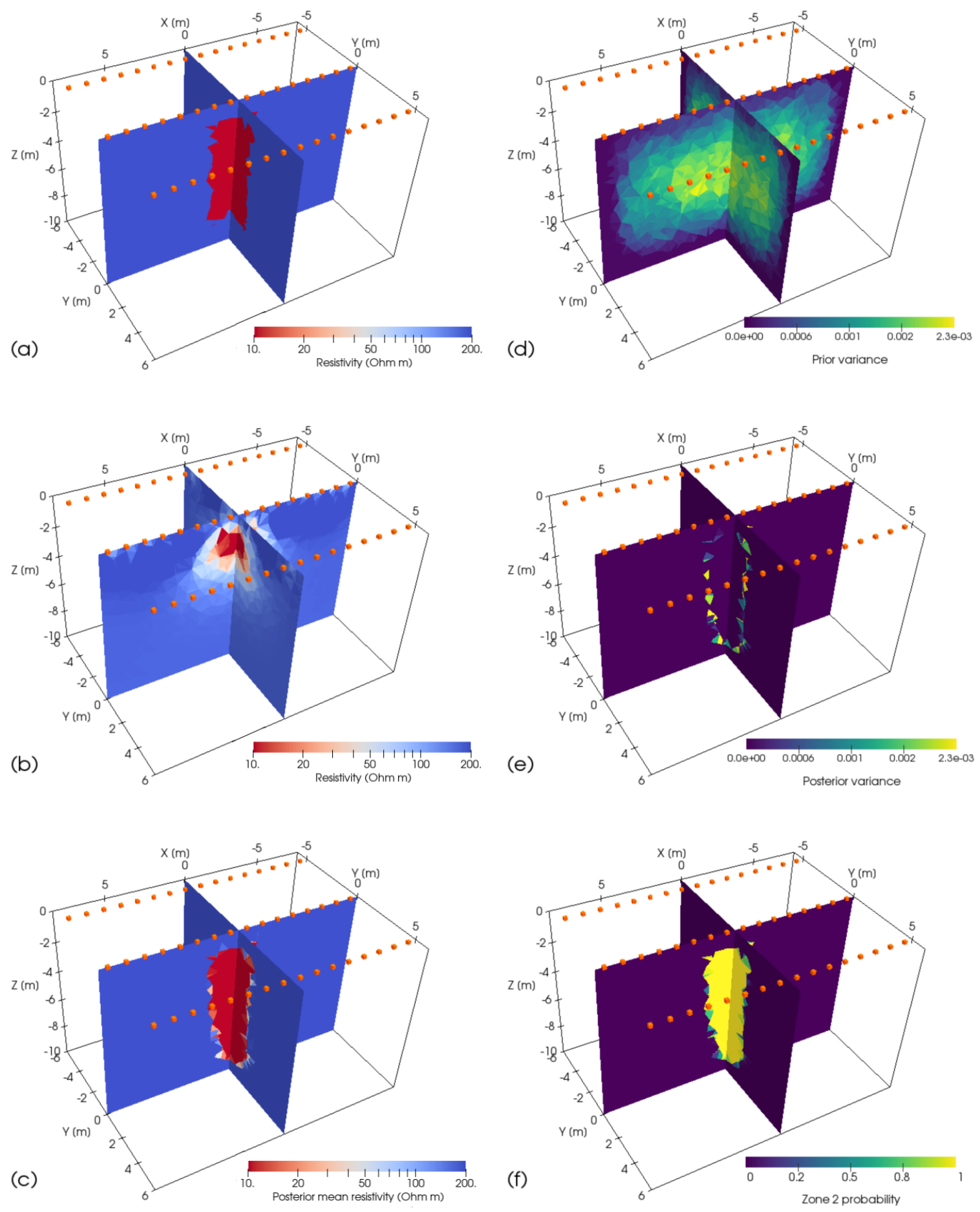

Figure 7. ERT inversion results of the 3D complex structure example: (a) The true resistivity model (b) The resistivity model estimated by smoothness-constrained inversion (c)The mean resistivity model estimated by EKI (d)The prior and (e) posterior variance map obtained by EKI (f) The posterior zone 2 probability map obtained by EKI. The location of the electrodes are marked as orange cubes. 


\subsection{Field examples}

\subsubsection{Borth peat bog, Wales, UK}

Comprised of $98 \%$ organic matter, peatlands are one of the largest reservoirs of carbon in the carbon cycle and are a major source of atmospheric methane. The existence of water logging and anaerobic condition slows down decomposition of plant materials, which in turn leads to the accumulation of peat. Estimating the extent and thickness are important to understand the hydrological and biogeochemical processes that occur in peatlands and geophysics has proved to be extremely useful in this regard (Slater \& Reeve 2002; Comas \& Slater 2004; Comas et al. 2004).

A series of field hydrogeophysical surveys and laboratory was conducted at Cors Fohno, a peat bog located at Borth in Wales, UK (52 $32^{\prime}$ N 04 00' W) (Asunbo 2007). GPR, ERT, IP and direct sampling measurements are taken at the site. In this example, we focus on a $2 \mathrm{~m}$ electrode spaced ERT survey conducted along a $94 \mathrm{~m}$ length of board walk at the site. The dataset consists of 397 dipole-dipole measurements with a dipole spacing of one electrode and up to 10 survey levels. The forward modelling grid comprises of 7904 rectangular cells while the EKI parameter grid consists of 300 and 60 cells ( $0.5 \mathrm{~m}$ spacing) in the $X$ and $Z$ directions respectively. A $5 \%$ measurement error is assumed for the inversions and a 2 zone formulation for EKI is used. The prior resistivity of zone 1 (background) and zone 2 are $[70,125] \Omega \mathrm{m}$ and $[140,200] \Omega m$ respectively.

Fig. 8a shows the results from smoothness-constrained inversion, which shows the resistivity decreases gradually with depth. Fig. 8b-d shows the results for EKI. Two almost perfectly horizontal zones are identified, which is expected from Fig. 8a and from other geophysical and core measurements taken at the site. The lower boundary is found to be about $4 \mathrm{~m}$ deep by EKI, which agrees with direct sampling results of Asunbo (2007), which shows the presence of peat down to $4-5 \mathrm{~m}$, underlained by dark organic-rich electrical conductive sediment down to 6.5-6.9m. This last boundary designates the peat's base which consists of blue (marine) clay. Fig. 8c-d shows that the high uncertainty areas (on zone membership) are at the boundaries of the zones and also at some locations at depth and on the right-hand-side of the panel. The higher complexity on the right-hand-side is also reported by Asunbo (2007).

In this example, convergence was achieved in 10 iterations. The computation was performed on an Intel i7 workstation (quad core) laptop and the time required for the entire inversion was less than 3 hours. 
(a)
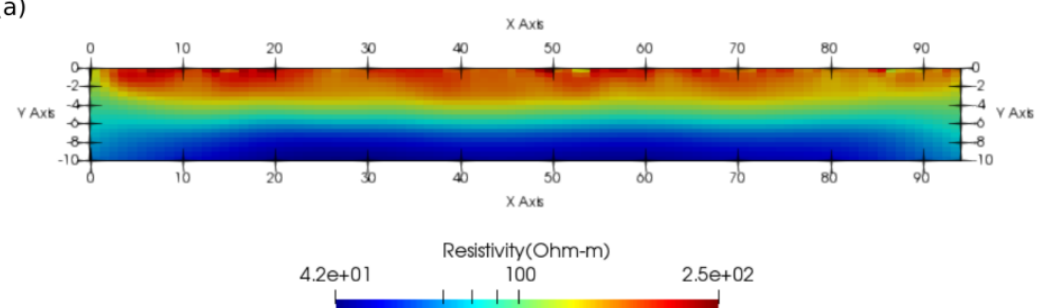

(b) $\quad$ mean $(\log 10($ resistivity in $\Omega \mathrm{m}))$
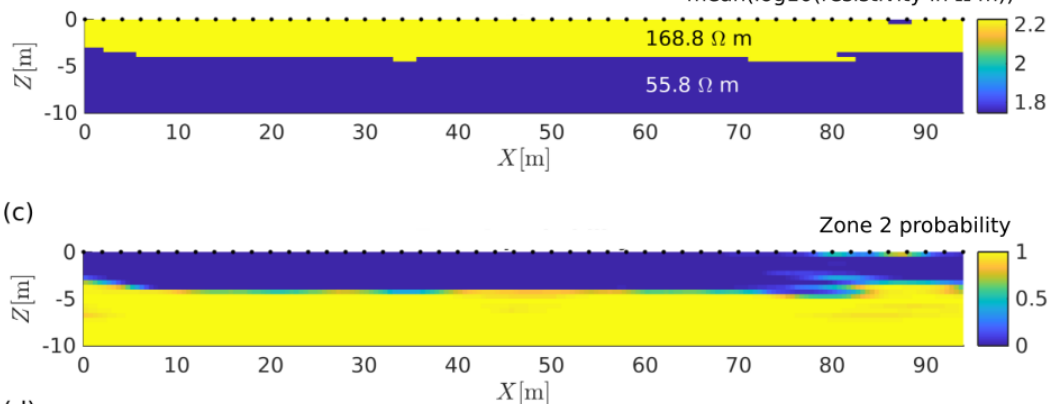

(d)

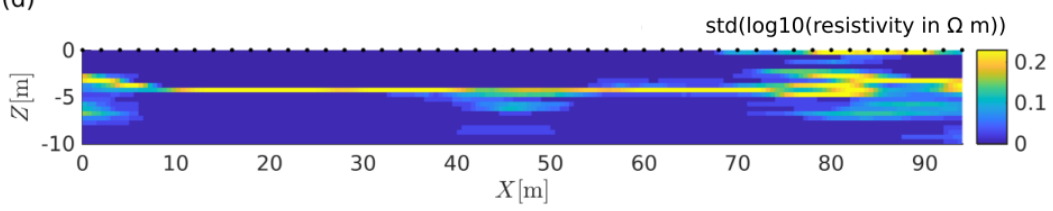

Figure 8. ERT inversion results of the Borth field example: (a) The resistivity model estimated by smoothness-constrained inversion reported in Asunbo (2007). The inset shows a photo of the ERT line. (b) The mean resistivity model estimated by EKI (c) The probability of a cell being in zone 2 estimated by EKI (d) The standard deviation map obtained by EKI. The location of the surface electrodes are marked as black circles in panels (b-d).

\subsubsection{Chenqi catchment hillslope, SW China}

The example data used here is from a survey conducted at Chenqi catchment in Guizhou Province, China in April 2017 (Cheng et al. 2019). An ERT line was deployed along a hillslope and the goal of the ERT survey was to identify the hydrostratigraphy of the hillslope in order to improve a hydrological conceptualization of runoff processes within the karstic catchment. The $2 \mathrm{D}$ profile consists of 48 electrodes and separated by about $5 \mathrm{~m}$. The exact position of the electrodes were surveyed and recorded. A dipole-dipole measurement configuration was used with dipole spacings of one, two, and three electrodes and up to 11 survey levels. A full set of reciprocal measurements of the data were taken for error analysis. The dataset consists of 1569 reciprocal measurements. A 5\% measurement error is assumed for the inversions. Fig. 9a shows the resistivity map obtained from smoothness constrained inversion, which shows a 
thin layer of conductive topsoil and several very smooth resistive regions. The latter can be interpreted as either horizontal layers or local inclusions.

We run inversion using EKI as a 3 zone problem and its results inform the choice of prior ranges of resistivity values based on smoothness constrained inversion results. For EKI, we use a parameter grid consisting of $1 \times 1$ grid cells spanning an area of $250 \mathrm{~m}$ by $140 \mathrm{~m}$ is used for parameter estimation. The prior resistivities of zone 1, zone 2 (background) and zone 3 are $[800,3000] \Omega \mathrm{m},[150,650] \Omega \mathrm{m}$ and $[10,100] \Omega \mathrm{m}$ respectively. The posterior mean estimates of the three zones are $1717.4 \Omega \mathrm{m}, 431.4 \Omega \mathrm{m}$ and $36.2 \Omega \mathrm{m}$ respectively. Since we allow zonal resistivity values to vary between realizations, Fig. 9e shows the histogram of posterior estimates of resistivity values for each zone. They do not vary greatly, as the structure of resistivity field is the main control of the ERT response. Fig. 9b shows an image of the mean resistivity estimates from EKI, which shows clearly the interfaces between zones. The bottom resistive zone is more extensive than estimated by smoothness-constrained inversion, while the two resistive zones at the upper left corner of the domain appears to be localized inclusions. EKI also captures a localized conductive zone at $X=170 \mathrm{~m}$, which does not appear in the smoothness-constrained inversion image. The resistivity maps obtained from EKI better captures the weathered features and soil infills that are expected in karstic systems like the ones at Chenqi, making the interpretation of the geometry of geological features from ERT images more intuitive. The high resistivity zone agrees with the occurrence of a very flat, slightly dipping mudstone in geological maps for the site (Cheng et al. 2019).

EKI also return the uncertainty estimates. Fig. 9c shows the probability map of a given cell to be a member of zone 2. A low value means a high probability of being a member of either zone 1 and 3. In the cropped region displayed in Fig. 9, there are no cells that has a non-zero probability of belonging to both zones 1 and 3 (or all three zones), making the zone 2 probability map a helpful way to visualize uncertainty. It appears for most cells that the zone 2 probability is either 0 or 1 . Alternatively, uncertainty can be visualized as a map of standard deviation across realizations of the posterior estimates (Fig. 9d). A high standard deviation is observed around interfaces, which is expected because in those cells there is significant probability for them to belong to more than one zone. This is expected in most ERT problems as the uncertainty within a structure is low and that along the interface of zones is high.

In this example, convergence was achieved in 29 iterations. The computation was performed on an Intel i7 workstation (quad core) laptop and the time required for the entire inversion was less than 5 hours, while a R2 inversion run takes less than two minutes. 

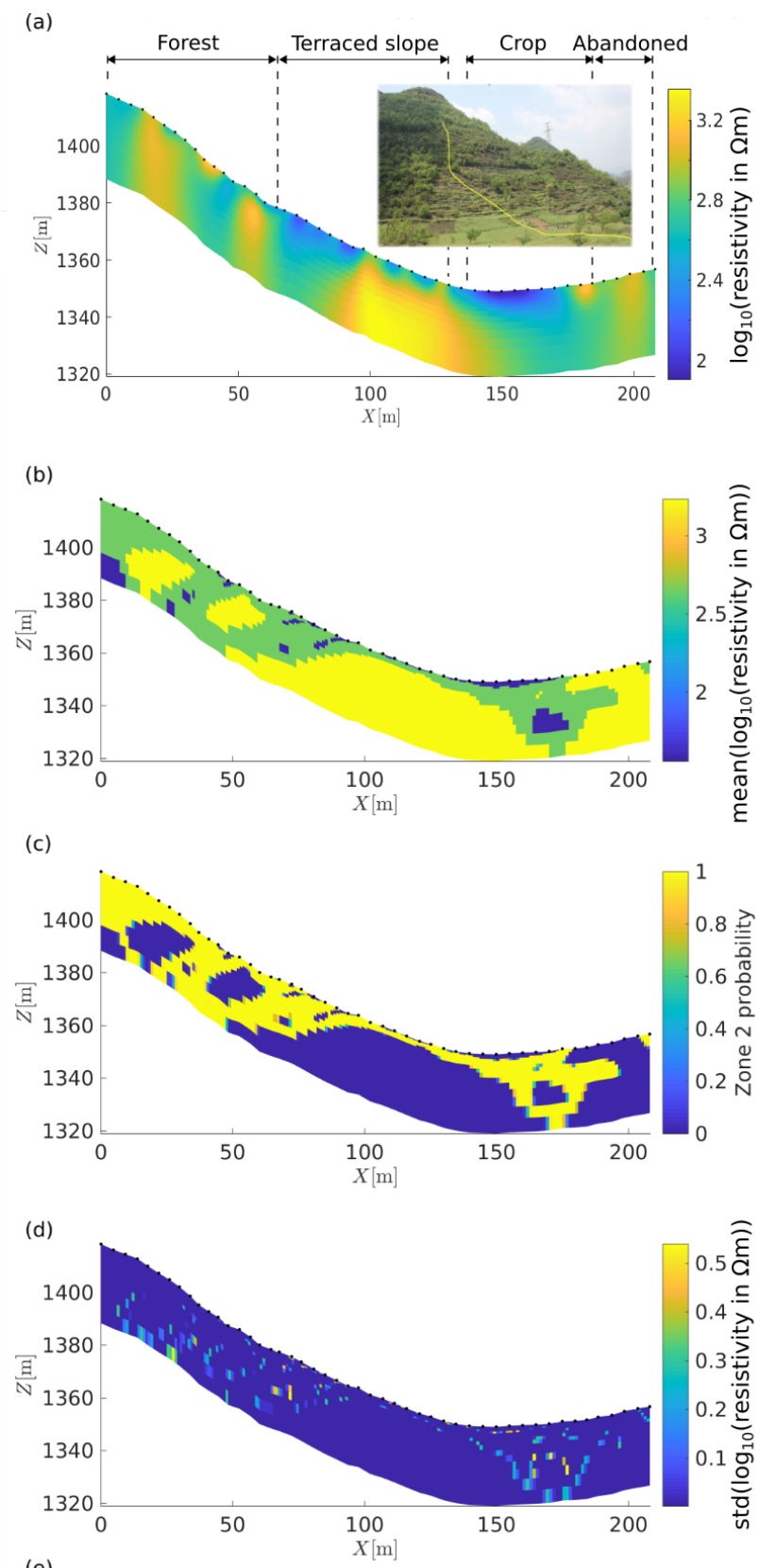

(e)

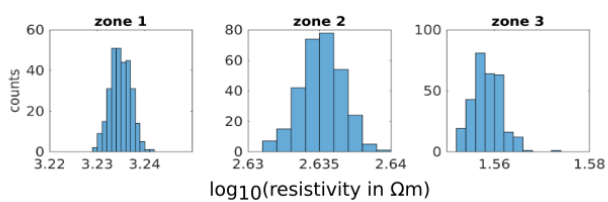

Figure 9. ERT inversion results of the Chenqi field example: (a) The resistivity model estimated by smoothness-constrained inversion. The inset shows a photo of the ERT line. (b) The mean resistivity model estimated by EKI (c) The probability of a cell being in zone 2 estimated by EKI (d) The standard deviation map obtained by EKI (e) A histogram of the EKI-estimated resistivity values of each zone across realizations. The location of the surface electrodes are marked as black circles. 


\subsubsection{Eggborough, Yorkshire, UK}

At Eggborough (National Grid Reference SE 570 232), ERT and GPR surveys were conducted in 1999 (Binley et al. 2002; Cassiani \& Binley 2005) to study the use of geophysical measurements for the parameterization of unsaturated hydraulic parameters. The data were later used to study the utility of joint inversion of ERT and GPR data (Linde et al. 2006; Bouchedda et al. 2012) and the influence of prior information on vadose zone parameters estimation in stochastic inversion (Scholer et al. 2011). Cores were collected at Eggborough to study petrophysical models for direct current resistivity and induced polarisation (Binley et al. 2005; Tso et al. 2019). In this example, we focus on the cross-borehole ERT survey for the panel R3-R4, where both surface and subsurface electrodes were used. The dataset consists of 6690 reciprocal measurements. The forward modelling grid consists of 3596 rectangular cells. The EKI parameter grid is $40 \mathrm{~m} \times 30 \mathrm{~m}$, comprised of 19200 grid cells that are uniformly spaced at $0.25 \mathrm{~m}$. The prior resistivity ranges of zone 1 , zone 2 (background) and zone 3 are $[10,80] \Omega \mathrm{m},[120,180] \Omega \mathrm{m}$ and $[200,400] \Omega \mathrm{m}$ respectively. A $5 \%$ measurement errors is assumed for the inversions.

Fig. 10a shows the resistivity estimates from a smoothness constrained inversion, using a 10 to 1 prescribed horizontal anisotropic ratio. Fig. 10b shows the mean resistivity map estimated by EKI, and the mean estimates of resistivity values of the three zones are 15.66, 108.52 , and $265.46 \Omega \mathrm{m}$. Without any of the information of the resistivity structure predefined, EKI was able to estimate a highly layered resistivity field. Its estimates also appears to be more realistic by showing irregular edges while the smoothness-constrained inversion shows almost horizontal layers. EKI also returns uncertainty estimates in Fig. 10c-d. The uncertainty appears to be particularly high at corners of zone boundaries.

Gamma logs and cross-hole radar measurements were also taken at Eggborough are compared against the EKI ERT results (Fig. 10b). Two shallow (4m to $5 \mathrm{~m}$ depth) high gamma counts region appear on R4 and E4 logs and appear to extend, albeit with a weaker signal, towards E3. This suggests a localized thickening of two clay rich (siltstone) units towards E4. Elevated moisture content in this zone (due to the vertical impedance of water movement caused by lower permeability) is also revealed by the low radar velocity between R3 and R4. These two shallow siltstone units align with the low resistivity zone in the inverted model; this feature does not extend to E3. The deeper high gamma counts region at $12 \mathrm{~m}$ also appears in the resistivity maps but is somewhat weaker because the moisture content is lower (as show in the radar profile). Finally, zone 1 at depths greater than $10 \mathrm{~m}$ correspond to a well-drained / coarser sandstone. The radar profile indicate dry zones around $12 \mathrm{~m}$ and $15 \mathrm{~m}$, 
which corresponds the zone 1 locations at R3 and R4. The comparison here illustrates the zones identified by EKI may not be the same geological unit throughout and care must be taken in their interpretation. Specifically, a EKI ERT inversion only define zones based on electrical signatures. It is expected that two or more facies of similar electrical signatures will be considered as the same zone by EKI.Andy will add interp.

The resistivity structure at Eggborough is complex and unlike a smoothness constrained inversion, EKI does not favor the simplest structure so it is possible to obtain multiple equally likely solutions. By re-running EKI using a different random seed for data noise, a alternative solution to Fig. 10 is obtained in Fig. 11. The solutions are very similar in terms of zonal resistivity values and they are both highly layered. However, the greater extent of the high resistivity zone $($ mean $=275.96 \Omega \mathrm{m})$ and a less resistive zone $2($ mean $=86.22 \Omega \mathrm{m})$ in Fig. 11 was compensated by the fact that Fig. 11 has only a small very low-resistivity zone (mean = $5.83 \Omega \mathrm{m})$ ). Both runs converge to the same misfit in the same number of iterations (Fig. 11d). The above implies some challenge to interpret EKI results but both cases honors the ERT data and other data at the site. If the resistivity of each zones are known, fixing them may help improve EKI performance.

In this example, convergence was achieved in 19 iterations. The computation was performed on an Intel i7 workstation (quad core) laptop and the time required for the entire inversion is less than 3 hours.

\section{DISCUSSION}

\subsection{Interpreting EKI results}

An ensemble Kalman inversion has been applied to ERT inversion, for the first time, to obtain resistivity images of the Earth's subsurface. It has been shown in previous theoretical studies that EKI can be treated as both a Gaussian approximation in sequential Monte Carlo approaches (Iglesias et al. 2018) and a regularizing, iterative optimizer that is an approximation to the Levenberg-Marquardt solution (Iglesias 2016; Muir \& Tsai 2020). This shows that EKI has preserved some for the strengths for both MCMC-type inversions and smoothnessconstrained inversion. The level set parameterization improves the performance of EKI in terms of both improving the well-posedness of the inverse problem (Iglesias et al. 2014) and allowing better estimation at interfaces (Iglesias et al. 2016).

In the presence of two or more materials with distinct resistivity ranges, it is well-known that the smoothness-constrained inversion will return a smoothed resistivity image and the 
(a)

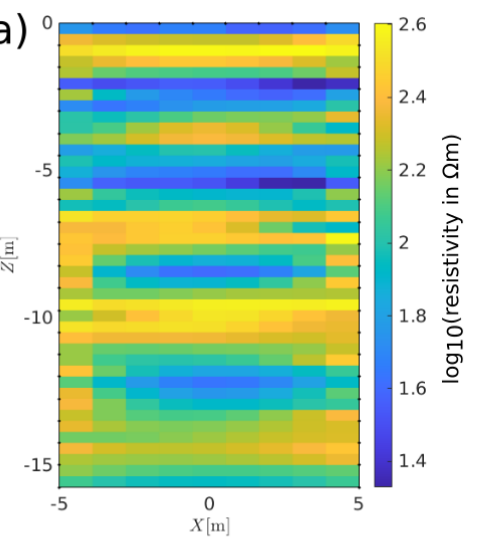

(c)

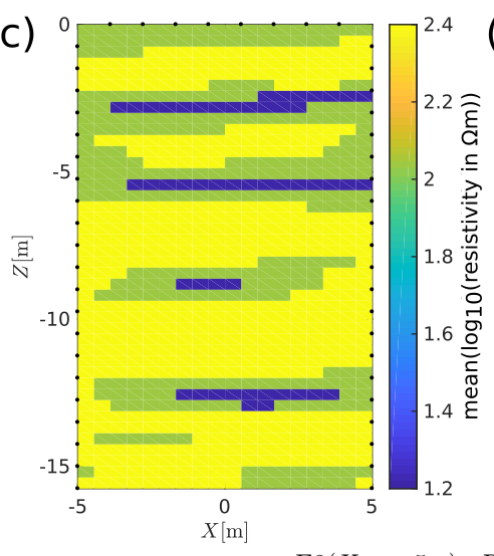

(f)

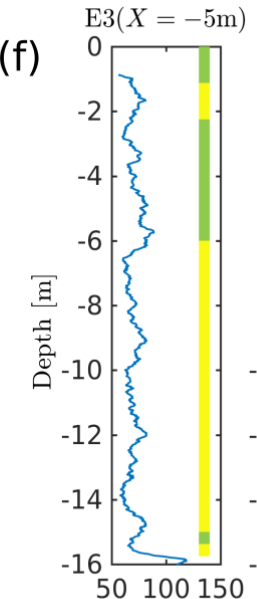

(d)
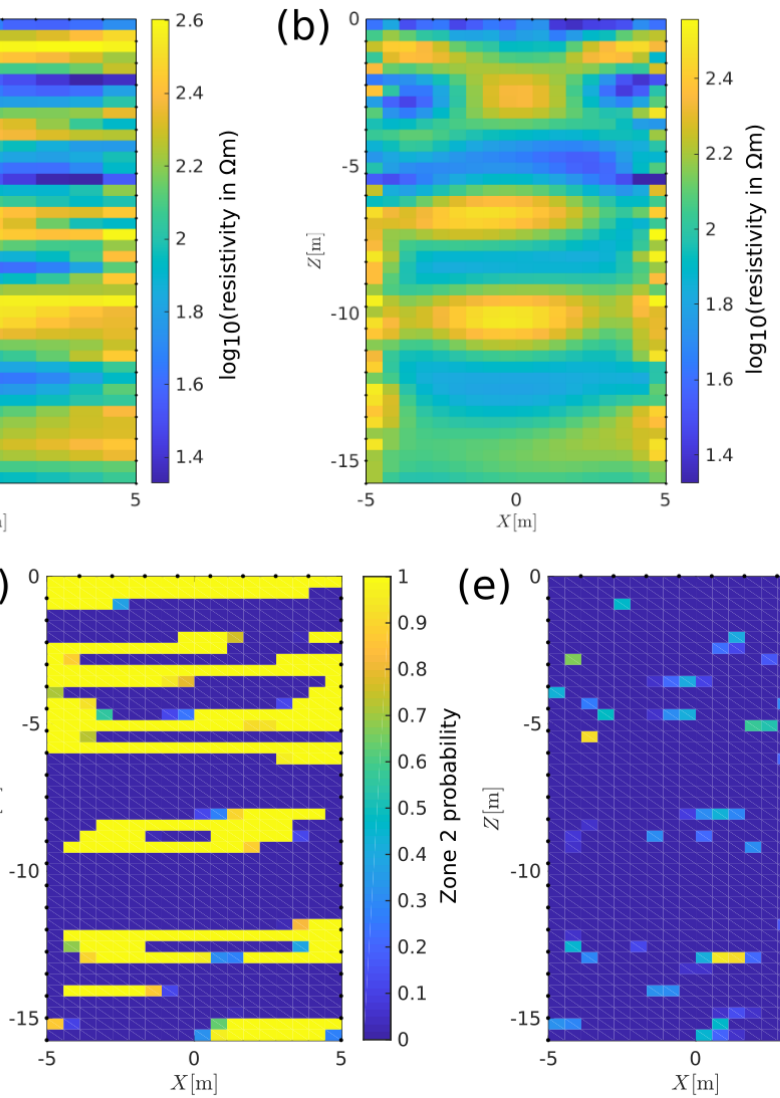

(e)
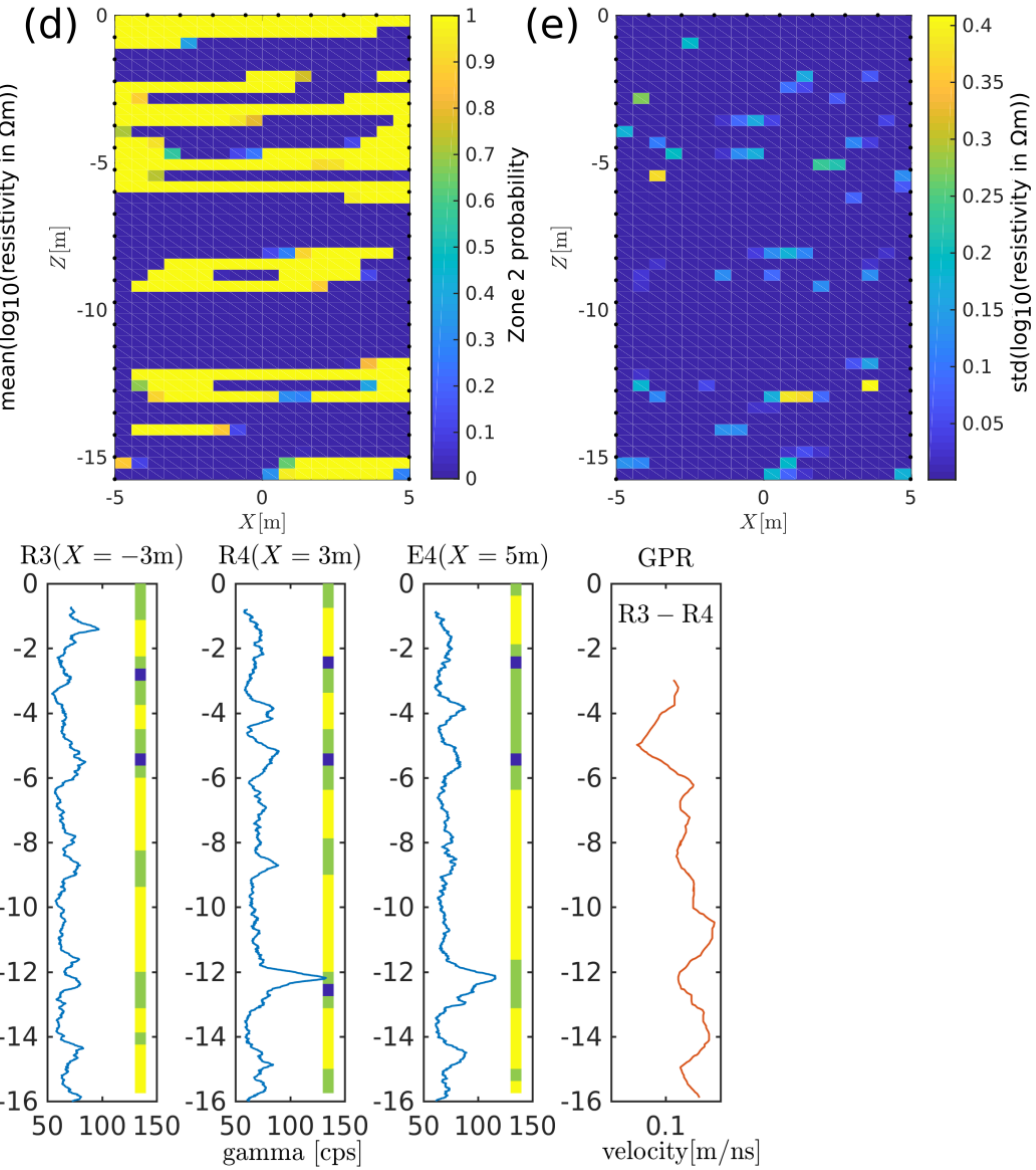

Figure 10. ERT inversion results of the Eggborough field example: The resistivity model estimated by smoothness-constrained inversion (a) with a 10:1 vertical anisotropy constraint and (b) without anisotropy constraint. (c) The mean resistivity model estimated by EKI (d) The probability of a cell being in zone 2 estimated by EKI (e) The standard deviation map obtained by EKI (f) The EKI mean resistivity from (b) is compared against gamma-ray logs and cross-borehole ground penetrating radar (GPR) results. The black circles in (a-e) denotes electrodes locations. Note that the surface electrodes extends for $5 \mathrm{~m}$ more in each direction. 

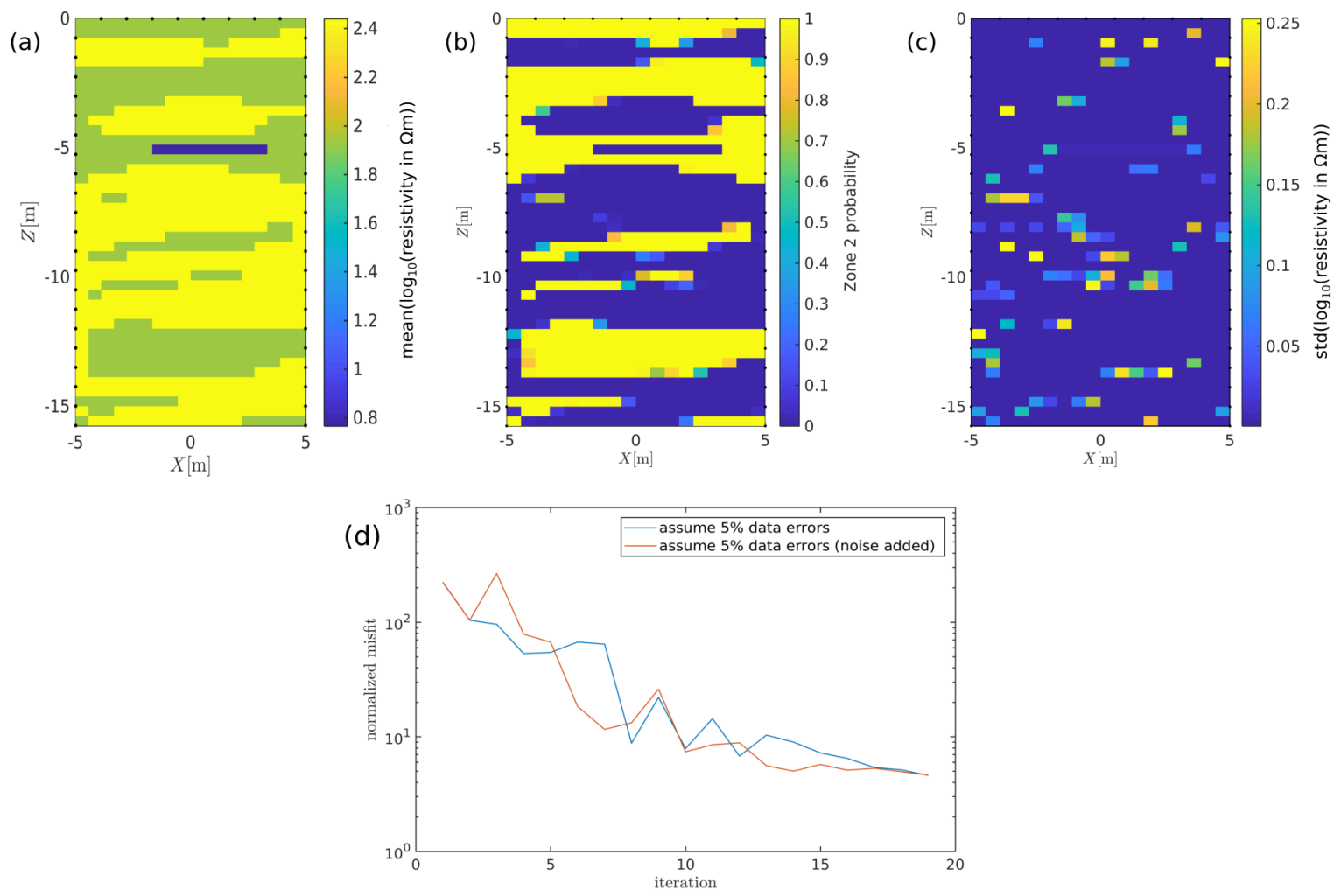

Figure 11. An alternative ERT inversion results of the Eggborough field example: (a) The mean resistivity model estimated by EKI (b) The probability of a cell being in zone 2 estimated by EKI (c) The standard deviation map obtained by EKI (d) The convergence behaviour of the two results are compared.

range of resistivity values are much smaller than the true range. This is because a sharp change in resistivity can be seen as a violation of assumption of the smoothness constrained inversion so the algorithm tries to restrict it as much as possible. Such features can limit the use of inverted resistivity values for further analysis (e.g. via petrophysical transforms). We observe from the results reported here that EKI tends not to suffer from these issues. The zone boundaries are well-recovered (even if they are arbitrarily shaped), the inverted resistivity values are in the same range as the actual ones, and the high uncertainty areas are not pre-dominated by their distance from the electrode array. Unlike smoothness constrained inversion, sharp interfaces are built into the prior distribution of prior models so the inverted resistivity image does not rapidly lose resolution when there is a sharp change in resistivity. EKI estimates is also affected by losing resolution away from electrodes, but less so than smoothness-constrained inversion. In our examples, we observe areas with pronounced artefacts in our EKI results, but they are located outside of the ERT imaging regions and are cropped out. 
EKI provides useful estimates of uncertainty by means of posterior variance maps and zonal probability maps. When repeating smoothness constrained inversion runs with perturbed data using Monte Carlo analysis, the uncertainty areas tend to be highest near the electrodes and decreases further away (Tso et al. 2019, 2017), which conveys more about the geometry of the electrode array than the uncertainty pattern of the ERT problem. This pattern is caused by low resolution in the smoothness-constrained inversion away from electrodes (as shown, for example, from a plot of the diagonal of the resolution matrix). These apparent low uncertainty zones are very misleading for uncertainty analysis (Tso et al. 2019; Tso 2019). The above issue can be solved partially by bootstrap sampling(Yang et al. 2014), nevertheless it is an uncertainty analysis on smooth images. Unlike smoothness constrained inversion, these maps appear to be more realistic and do not show the misleading correlation between proximity to electrodes and variance of inverted resistivity. Nevertheless, the posterior uncertainty tends to be very low away from the interfaces (or zonal membership very close to 0 or 1 ). It is important to note that EKI uses a small ensemble and each of the ensemble member move towards the misfit minima (and we have not specified anything to keep them apart). So the value of the uncertainty estimates should not be treated as the full parameter uncertainty. Such considerations should be taken account when interpreting EKI uncertainty estimates, especially if they are propagated to subsequent analysis (e.g. they may need to be inflated). Nevertheless, the uncertainty pattern returned by EKI is very helpful to guide interpretation and further analysis.

The prior resistivity ranges of the different zones do not seem to show a great impact to the EKI results. However, resistivity ranges between zones should not overlap in order for the level set parametrization to work properly. We set the tuning factor to 30 in all our examples in this work - its selection is critical to the performance of the method. Our method also works best when sharp interfaces are expected and there are known contrasts between the resistivity of the two zones. It is possible that it will mistake a gradual change in resistivity for an abrupt one. Precautions should also be taken when interpreting low-uncertainty regions returned by EKI. For example, when EKI estimates gives low variance and zonal probability equals 0 or 1 . It only represents the uncertainty given the inverse problem setup (e.g. assumptions or number of zones and prior resistivity ranges). These factors needs to be taken into consideration when interpreting (and propagating) EKI uncertainty maps. 


\subsection{Comparison with MCMC methods}

As mentioned in the introduction, smoothness-constrained inversions are not suitable for uncertainty quantification because it tends to show low sensitivity areas as low variance (i.e. uncertainty) areas. Therefore, such uncertainty estimates are not helpful when used to propagate uncertainties in subsequent analysis. While there have been efforts to mitigate such issues by post-possessing smoothness-constrained inversion results, they only highlight potentially high uncertainty areas qualitatively.

In contrast, MCMC inversion has been considered as the gold standard for uncertainty modelling in many disciplines including hydrogeophysics. Many consider it as fully Bayesian and has advantages such as thoroughly sampling the parameter space and allowing estimation of posterior pdfs with multiple modes. However, its very high computing effort (i.e. normally requiring $10^{3}-10^{6}$ model runs) prohibits its use in most practical ERT applications.

In the trans-dimensional MCMC ERT inversion of Galetti \& Curtis (2018), "uncertainty loops" (i.e. high uncertainty bands) have been observed near the interface between zones. Since their method obtains a very detailed full posterior pdf, these "uncertainty loops" (Galetti et al. 2015) are areas where the posterior pdf is flat and multiple modes are present. The loops are helpful to visualize uncertainty in parameter estimation and they have not been reported using other methods. Although the EKI cannot obtain the full posterior pdf as in Galetti \& Curtis (2018), we observe regions of high uncertainty (i.e. variance) around the zonal interfaces in our synthetic examples. This shows that the EKI method can offer a decent approximation of the extent of "uncertainty loops".

\subsection{Applicability and potential extensions}

In the last couple of years, EKI or ensemble Kalman filter (EnKF) methods have been used increasingly in hydrogeophysics. For example, Tso et al. (2020) used ensemble smoother for multiple data assimilation (ES-MDA, Emerick \& Reynolds (2013)) to estimates leak parameters for field-scale leak events from ERT data. Kang et al. $(2018,2019)$ used EnKF to estimate DNAPL distribution from ERT data of sandbox experiments. Claes et al. (2020) used ES-MDA to calibrate zonal $\mathrm{K}$ values of a watershed model from ERT data. However, EKI/ES-MDA has not been used for ERT or geophysical inversion or imaging before (although derived ERT data such as travel times or spatial moments have been used). Without the level-set parameterization, many geophysical inversion problems would have too many unknowns to be practical. However, as highlighted above that EKI/ES-MDA are well suited for solving coupled inverse 
problems thanks to its derivative-free formulation. EKI with level set formulation may open up future opportunities for coupled hydrogeophysical inversion of heterogeneous fields.

As proposed by Cardiff \& Kitanidis (2009) in the groundwater/hydrogeophysics literature, the level set parameterization is an extensible framework for facies detection that is suitable for joint or coupled inversion (e.g. hydraulic head and seismic veolcity) and uncertainty quantification. However, the uptake has been slow and it has not been used in practical ERT problems (although they have been included in theoretical work (e.g. Aghasi et al. 2011; Chada et al. 2018; Chung et al. 2005) ). As shown here, EKI significantly reduces the computation cost and thus lower the barrier for Bayesian inversion and uncertainty quantification. The use of EKI as a parameter estimation method makes it computationally much less intensive than other level set methods previously reported in the hydrogeophysics literature (Cardiff \& Kitanidis 2009). EKI is also much very flexible to incorporate data from different modalities due to the "black-box" nature (i.e. derivative-free) of its inversion formulation. It has been increasingly recognized that one can constrain a smoothness constrained inversion by geostatistical data (by modifying the a priori model covariance matrix) (e.g. Hermans et al. 2012). As shown in Muir \& Tsai (2020) recently in their deep-earth seismic work, this can be done within the EKI but with much greater flexibility (see also comments in section 2.4).

A straightforward extension of this work is to consider its best formulation in time-lapse ERT problems. Difference inversion (Labrecque \& Yang 2001) has been a standard for smoothness constrained inversion of time-lapse ERT surveys. Future studies should verify whether the current EKI formulation is suitable for time-lapse ERT studies and whether a difference inversion can further improve the method for time-lapse ERT use. Likewise, the examples considered here contains extensive features (e.g. inclusions, layers). Given the flexibility of its parameterization, it will be worth considering its use in imaging discrete features (e.g. planar hydraulic fractures) (Wu et al. 2019).

Smoothness constrained inversion will probably continue to be the most popular method for ERT inversion. It is a very helpful tool to evaluate ERT data in the first instance. However, the EKI method described here has significantly lower the barrier of entry for performing uncertainty quantification and reliably identify zonal interfaces for ERT inversion. Its use should be encouraged to improve the interpretability of ERT.

\section{CONCLUSIONS}

We have described an efficient method, based on ensemble Kalman inversion with level set parametrization, for ERT inversion that is suitable for uncertainty quantification and imaging 
(potentially discontinuous) multi-scale features. In particular, our field examples show that it can handle arbitrarily shaped layers and inclusions (without specifying anisotropy). Our method is efficient enough for solving $2 \mathrm{D}$ problems in a personal computer and 3D problems in a small computer cluster. Its computational cost is a fraction of that of MCMC methods, while also circumventing apparent issues with uncertainty quantification when using smoothness-constrained inversions. Importantly, it produces uncertainty estimates that are helpful for interpretation and are fit-for-purpose for uncertainty propagation. The level set parametrization we use provides a flexible framework to handle spatially varying changes in the resistivity field and it does not require strong assumptions on the length scales or structure of the resistivity fields. Such method contributes to the continuing effort to improve resistivity imaging of material interfaces and to broaden the use of uncertainty quantification in ERT applications. It is our intention that it will serve as a useful tool in a wide range of hydrogeophysics applications.

\section{AUTHOR CONTRIBUTION STATEMENT}

MI conceived the research, wrote the methods section and the codes for EKI with level set parametrization, and ran the 3D experiment. MT drafted the rest of the manuscript, adapted the codes for ERT and ran all the 2D experiments with R2. MT and AB designed the test cases. All authors provided feedback to the design and edited the manuscript.

\section{ACKNOWLEDGMENTS}

This article is published with the permission of the executive director of the BGS. Michael Tso was supported by a Lancaster University PhD studentship and a Nuclear Decommissioning Authority (UK) bursary. We thank Oliver Kuras (BGS) for his support to kickstart this work.

We thank Tim Johnson for help with using E4D, and University of Nottingham and BGS for access to computing resources. The field data used originated from Lancaster-NERC grants: NE/N007409/1 (Chenqi); NE/F004958/1 (Borth); NER/A/S/2001/01175 (Eggborough). Field assistance from Lancaster researchers and students Qinbo Cheng (Chenqi), Adeola Asunbo \& Nick Kettridge (Borth) and Peter Winship (Eggborough) is acknowledged. Example codes to implement the method is available via the corresponding author. 


\section{REFERENCES}

Aghasi, A., Kilmer, M., \& Miller, E. L., 2011. Parametric level set methods for inverse problems, SIAM Journal on Imaging Sciences, 4(2), 618-650.

Andersen, K. E., Brooks, S. P., \& Hansen, M. B., 2003. Bayesian inversion of geoelectrical resistivity data, Journal of the Royal Statistical Society: Series B (Statistical Methodology), 65(3), 619-642.

Arridge, S., Maass, P., Öktem, O., \& Schönlieb, C.-B., 2019. Solving inverse problems using datadriven models, Acta Numerica, 28, 1-174.

Asunbo, A., 2007. Improving the hydrological characterization of peatlands using geophysics, Master's thesis, Lancaster University.

Binley, A., 2015. Tools and techniques: Electrical methods.

Binley, A., Cassiani, G., Middleton, R., \& Winship, P., 2002. Vadose zone flow model parameterisation using cross-borehole radar and resistivity imaging, Journal of Hydrology, 267(3-4), 147-159.

Binley, A., Slater, L. D., Fukes, M., \& Cassiani, G., 2005. Relationship between spectral induced polarization and hydraulic properties of saturated and unsaturated sandstone, Water Resources Research, 41, W12417.

Blanchy, G., Saneiyan, S., Boyd, J., McLachlan, P., \& Binley, A., 2020. ResIPy, an intuitive open source software for complex geoelectrical inversion/modeling, Computers \& Geosciences, 137, 104423.

Bouchedda, A., Chouteau, M., Binley, A., \& Giroux, B., 2012. 2-D joint structural inversion of crosshole electrical resistance and ground penetrating radar data, Journal of Applied Geophysics, 78, $52-67$.

Bouchedda, A., Bernard, G., \& Gloaguen, E., 2017. Constrained electrical resistivity tomography Bayesian inversion using inverse Matérn covariance matrix, Geophysics, 82(3), E129-E141.

Camporese, M., Cassiani, G., Deiana, R., Salandin, P., \& Binley, A., 2015. Coupled and uncoupled hydrogeophysical inversions using ensemble Kalman filter assimilation of ERT-monitored tracer test data, Water Resources Research, 51(5), 3277-3291.

Cardiff, M. \& Kitanidis, P. K., 2009. Bayesian inversion for facies detection: An extensible level set framework, Water Resources Research, 45(10), 1-15.

Cassiani, G. \& Binley, A., 2005. Modeling unsaturated flow in a layered formation under quasi-steady state conditions using geophysical data constraints, Advances in Water Resources, 28(5), 467-477.

Chada, N. K., Iglesias, M. A., Roininen, L., \& Stuart, A. M., 2018. Parameterizations for ensemble Kalman inversion, Inverse Problems, 34(5), 055009.

Chambers, J. E., Wilkinson, P. B., Uhlemann, S., Sorensen, J. P. R., Roberts, C., Newell, A. J., Ward, W. O. C., Binley, A., Williams, P. J., Gooddy, D. C., Old, G., \& Bai, L., 2014. Derivation of lowland riparian wetland deposit architecture using geophysical image analysis and interface detection, Water Resources Research, 50(7), 5886-5905.

Chan, T. F. \& Tai, X.-C., 2004. Level set and total variation regularization for elliptic inverse problems 
with discontinuous coefficients, Journal of Computational Physics, 193(1), 40-66.

Chen, C. \& Zeng, L., 2015. Using the level set method to study the effects of heterogeneity and anisotropy on hyporheic exchange, Water Resources Research, 51(5), 3617-3634.

Chen, X., Hammond, G. E., Murray, C. J., Rockhold, M. L., Vermeul, V. R., \& Zachara, J. M., 2013. Application of ensemble-based data assimilation techniques for aquifer characterization using tracer data at Hanford 300 area, Water Resources Research, 49(10), 7064-7076.

Cheng, Q., Chen, X., Tao, M., \& Binley, A., 2019. Characterization of karst structures using quasi-3d electrical resistivity tomography, Environmental Earth Sciences, 78(9).

Chung, E. T., Chan, T. F., \& Tai, X.-C., 2005. Electrical impedance tomography using level set representation and total variational regularization, Journal of Computational Physics, 205(1), 357372.

Claes, N., Paige, G., Grana, D., \& Parsekian, A., 2020. Parameterization of a Hydrologic Model with Geophysical Data to Simulate Observed Subsurface Return Flow Paths, Vadose Zone Journal, 19(1), e20024.

Comas, X. \& Slater, L., 2004. Low-frequency electrical properties of peat, Water Resources Research, $\mathbf{4 0}(12)$.

Comas, X., Slater, L., \& Reeve, A., 2004. Geophysical evidence for peat basin morphology and stratigraphic controls on vegetation observed in a northern peatland, Journal of Hydrology, 295(1), $173-184$.

de Pasquale, G., Linde, N., Doetsch, J., \& Holbrook, W. S., 2019. Probabilistic inference of subsurface heterogeneity and interface geometry using geophysical data, Geophysical Journal International.

Dunlop, M. M., Iglesias, M. A., \& Stuart, A. M., 2017. Hierarchical bayesian level set inversion, Statistics and Computing, 27(6), 1555-1584.

Emerick, A. A. \& Reynolds, A. C., 2013. Ensemble smoother with multiple data assimilation, Computers and Geosciences, 55, 3-15.

Englert, A., Kemna, A., feng Zhu, J., Vanderborght, J., Vereecken, H., \& Yeh, T.-C. J., 2016. Comparison of smoothness-constrained and geostatistically based cross-borehole electrical resistivity tomography for characterization of solute tracer plumes, Water Science and Engineering, 9(4), $274-$ 286.

Galetti, E. \& Curtis, A., 2018. Transdimensional Electrical Resistivity Tomography, Journal of Geophysical Research: Solid Earth.

Galetti, E., Curtis, A., Meles, G. A., \& Baptie, B., 2015. Uncertainty Loops in Travel-Time Tomography from Nonlinear Wave Physics, Physical Review Letters, 114(14), 148501.

Hermans, T. \& Irving, J., 2017. Facies discrimination with electrical resistivity tomography using a probabilistic methodology: Effect of sensitivity and regularization, Near Surface Geophysics, 15, $13-25$.

Hermans, T., Vandenbohede, A., Lebbe, L., Martin, R., Kemna, A., Beaujean, J., \& Nguyen, F., 
2012. Imaging artificial salt water infiltration using electrical resistivity tomography constrained by geostatistical data, Journal of Hydrology, 438-439, 168-180.

Iglesias, M., Lu, Y., \& Stuart, A., 2016. A Bayesian level set method for geometric inverse problems, Interfaces and Free Boundaries, 18(2), 181-217.

Iglesias, M., Park, M., \& Tretyakov, M. V., 2018. Bayesian inversion in resin transfer molding, Inverse Problems, 34(10), 105002.

Iglesias, M. A., 2016. A regularizing iterative ensemble Kalman method for PDE-constrained inverse problems, Inverse Problems, 32(2), 025002.

Iglesias, M. A. \& Yang, Y., 2020. Adaptive regularisation for ensemble Kalman inversion with applications to non-destructive testing and imaging, Submitted.

Iglesias, M. A., Lin, K., \& Stuart, A. M., 2014. Well-posed Bayesian geometric inverse problems arising in subsurface flow, Inverse Problems, 30(11), 114001.

Irving, J. \& Singha, K., 2010. Stochastic inversion of tracer test and electrical geophysical data to estimate hydraulic conductivities, Water Resources Research, 46(11), W11514.

Johnson, T. C., Versteeg, R. J., Ward, A., Day-Lewis, F. D., \& Revil, A., 2010. Improved hydrogeophysical characterization and monitoring through parallel modeling and inversion of time-domain resistivity and induced-polarization data, Geophysics, 75(4), WA27.

Johnson, T. C., Versteeg, R. J., Rockhold, M., Slater, L. D., Ntarlagiannis, D., Greenwood, W. J., \& Zachara, J., 2012. Characterization of a contaminated wellfield using 3d electrical resistivity tomography implemented with geostatistical, discontinuous boundary, and known conductivity constraints, Geophysics, 77(6), EN85-EN96.

Kaipio, J. \& Somersalo, E., 2005. Statistical and Computational Inverse Problems, Springer, Dordrecht.

Kang, X., Shi, X., Deng, Y., Revil, A., Xu, H., \& Wu, J., 2018. Coupled hydrogeophysical inversion of DNAPL source zone architecture and permeability field in a 3D heterogeneous sandbox by assimilation time-lapse cross-borehole electrical resistivity data via ensemble Kalman filtering, Journal of Hydrology, 567, 149-164.

Kang, X., Shi, X., Revil, A., Cao, Z., Li, L., Lan, T., \& Wu, J., 2019. Coupled hydrogeophysical inversion to identify non-Gaussian hydraulic conductivity field by jointly assimilating geochemical and time-lapse geophysical data, Journal of Hydrology.

Kitanidis, P. K., 2015. Three-dimensional ERT imaging by the geostatistical approach, in American Geophysical Union Fall Meeting, San Francisco, California.

Labrecque, D. J. \& Yang, X., 2001. Difference inversion of ERT data: a fast inversion method for 3-D in situ monitoring, Journal of Environmental 6 Engineering Geophysics, 6(83), 83-89.

Linde, N., Binley, A., Tryggvason, A., Pedersen, L. B., \& Revil, A., 2006. Improved hydrogeophysical characterization using joint inversion of cross-hole electrical resistance and ground-penetrating radar traveltime data, Water Resources Research, 42(12), WR005131. 
Linde, N., Ginsbourger, D., Irving, J., Nobile, F., \& Doucet, A., 2017. On uncertainty quantification in hydrogeology and hydrogeophysics, Advances in Water Resources, 110, 166-181.

Lindgren, F., Rue, H., \& Lindström, J., 2011. An explicit link between Gaussian fields and Gaussian Markov random fields: the stochastic partial differential equation approach, Journal of the Royal Statistical Society: Series B (Statistical Methodology), 73(4), 423-498.

Loke, M., Acworth, I., \& Dahlin, T., 2003. A comparison of smooth and blocky inversion methods in 2d electrical imaging surveys, Exploration Geophysics, 34(3), 182-187.

Muir, J. B. \& Tsai, V. C., 2020. Geometric and level set tomography using ensemble Kalman inversion, Geophysical Journal International, 220, 967-980.

Nguyen, F., Kemna, A., Robert, T., \& Hermans, T., 2016. Data-driven selection of the minimumgradient support parameter in time-lapse focused electric imaging, Geophysics, 81(1), A1-A5.

Nussbaumer, R., Linde, N., Mariethoz, G., \& Holliger, K., 2019. Simulation of fine-scale electrical conductivity fields using resolution-limited tomograms and area-to-point kriging, Geophysical Journal International, 218(2), 1322-1335.

Ramirez, A. L., Nitao, J. J., Hanley, W. G., Aines, R., Glaser, R. E., Sengupta, S., Dyer, K. M., Hickling, T., \& Daily, W. D., 2005. Stochastic inversion of electrical resistivity changes using a Markov Chain Monte Carlo approach, Journal of Geophysical Research: Solid Earth, 110, B02101.

Roininen, L., Lasanen, S., Orispää, M., \& Särkkä, S., 2014. Sparse approximations of fractional Mat $\backslash$ 'ern fields.

Sassen, D. S., Hubbard, S. S., Bea, S. A., Chen, J., Spycher, N., \& Denham, M. E., 2012. Reactive facies: An approach for parameterizing field-scale reactive transport models using geophysical methods, Water Resources Research, 48, 1-20.

Scholer, M., Irving, J., Binley, A., \& Holliger, K., 2011. Estimating vadose zone hydraulic properties using ground penetrating radar: The impact of prior information, Water Resources Research, 47(10), $1-14$.

Si, H., 2015. TetGen, a Delaunay-Based Quality Tetrahedral Mesh Generator, ACM Transactions on Mathematical Software, 41(2), 1-36.

Slater, L. \& Binley, A., 2003. Evaluation of permeable reactive barrier (PRB) integrity using electrical imaging methods, Geophysics, 68(3), 911-921.

Slater, L. D. \& Reeve, A., 2002. Investigating peatland stratigraphy and hydrogeology using integrated electrical geophysics, Geophysics, 67(2), 365-378.

Song, X., Chen, X., Ye, M., Dai, Z., Hammond, G., \& Zachara, J. M., 2019. Delineating Facies Spatial Distribution by Integrating Ensemble Data Assimilationand Indicator Geostatistics With Level-Set Transformation, Water Resources Research, p. 2018WR023262.

Stuart, A., 2010. Inverse problems: a Bayesian perspective, in Acta Numerica, vol. 19, pp. $451-559$. Tso, C.-H. M., Kuras, O., Wilkinson, P. B., Uhlemann, S., Chambers, J. E., Meldrum, P. I., Graham, J., Sherlock, E. F., \& Binley, A., 2017. Improved characterisation and modelling of measurement 
errors in electrical resistivity tomography (ERT) surveys, Journal of Applied Geophysics, 146, 103119.

Tso, C.-H. M., Kuras, O., \& Binley, A., 2019. On the Field Estimation of Moisture Content Using Electrical Geophysics: The Impact of Petrophysical Model Uncertainty, Water Resources Research, 55(8), 7196-7211.

Tso, C.-H. M., Johnson, T. C., Song, X., Chen, X., Kuras, O., Wilkinson, P., Uhlemann, S., Chambers, J. E., \& Binley, A., 2020. Integrated hydrogeophysical modelling and data assimilation for geoelectrical leak detection, Journal of Contaminant Hydrology.

Tso, M., 2019. Enhancing the information content of geophysical data for nuclear site characterisation, Ph.D. thesis, Lancaster University.

Wainwright, H. M., Chen, J., Sassen, D. S., \& Hubbard, S. S., 2014. Bayesian hierarchical approach and geophysical data sets for estimation of reactive facies over plume scales, Water Resources Research, 50(6), 4564-4584.

Ward, W., Wilkinson, P., Chambers, J., Oxby, L., \& Bai, L., 2014. Distribution-based fuzzy clustering of electrical resistivity tomography images for interface detection, Geophysical Journal International, 197(1), 310-321.

Wu, H., Fu, P., Yang, X., Morris, J. P., Johnson, T. C., Settgast, R. R., \& Ryerson, F. J., 2019. Accurate imaging of hydraulic fractures using templated electrical resistivity tomography, Geothermics, $81,74-87$.

Yang, X., Chen, X., Carrigan, C. R., \& Ramirez, A. L., 2014. Uncertainty quantification of CO2 saturation estimated from electrical resistance tomography data at the Cranfield site, International Journal of Greenhouse Gas Control, 27, 59-68.

Yeh, T.-C. J., Liu, S., Glass, R. J., Baker, K., Brainard, J. R., Alumbaugh, D., \& LaBrecque, D., 2002. A geostatistically based inverse model for electrical resistivity surveys and its applications to vadose zone hydrology, Water Resources Research, 38(12), WR001204.

Zhou, H., Gómez-Hernández, J. J., \& Li, L., 2014. Inverse methods in hydrogeology: Evolution and recent trends, Advances in Water Resources, 63, 22-37. 